\title{
Discussions on Hirsch Conjecture and The Existence of Strongly Polynomial: Time Simplex Variants
}

\author{
Pei-Zhuang Wang
}

Received: 5 November 2012 / Revised: 16 January 2013 / Accepted: 20 April 2013 /

Published online: 23 April 2014

(C) Springer-Verlag GmbH Berlin Heidelberg 2014

\begin{abstract}
Based on the theory of cone-cutting, a preliminary analysis for Hirsch conjecture is made in the paper. The author indirectly proves a related assembling conjecture under condition 'Fully cuts' and suggests a relaxed conjecture to deny the existence of super polynomial diameters on LP polytopes. An algorithm named Gradient Falling searching the dual optimal point $\boldsymbol{y}^{*}$ in dual space $Y$ from a given feasible point is presented, which traces a shortest path within the dual feasible region $D$, the author guesses, it may be an example for the existence of strongly polynomial-times LP algorithms.
\end{abstract}

Keywords Linear programming $\cdot$ Simplex $\cdot$ Strongly polynomial algorithm $\cdot$ Hirsch conjecture $\cdot$ Cone-cutting

\section{Mathematics Subject Classification 90C05}

\section{Introduction}

The simplex-pivot developed by Dantzig [1] is an elegant mathematical algorithm providing quite an efficient method for most practical LP applications, especially, for data mining [2]. Even so, we are anxious about the polynomial-time solvability of the simplex variants. Although the essential progress on the polynomial-time solv-

\footnotetext{
P.-Z. Wang $(\varangle)$

College of Science, Liaoning Engineering Technology Univ.,

Liaoning 123000, Fuxin, China

e-mail: peizhuangw@126.com

P.-Z. Wang

Research Center of Fictitious Economy and Data Science,

Chinese Academy of Sciences, Beijing 100080, China
} 
ability has been taken by the ellipsoid methods and interior-point techniques since the 1970s, the run time's complexity of them is only qualified as 'weak polynomial'. No algorithm has yet been demonstrated as a strong polynomial-time performance depending only on the number of constraints $m$ and the number of variables $n$. Exploration and development of such algorithms will have great theoretical interest and practical needs, which has been cited by Stephen Smale as one among the 18 greatest unsolved problems of the twenty-first century [3].

G. Hirsch wrote a letter to Dantzig in 1957 about the feature of LP polytopes, which could be roughly understood as a convex region $P$ cut by a group of constraint facets in the solution-space $X=R^{n}$. Since the simplex-pivot keeps the prime feasible basic solution from one vertex to another vertex moving along the edges of $P$, the performance of a simplex algorithm traces a track moving from original prime basic solution $\underline{\boldsymbol{x}}^{0}$ to the prime optimal point $\underline{\boldsymbol{x}}^{*}$, and this is so called the edge-following algorithm. Even though Dantzig's original simplex is not a polynomial algorithm disproved by an anti-example of non-degenerated exponential performance, we can not say that no strong polynomial-time edge-following algorithm exists. A simplexvariant algorithm is possible to avoid the worse tracks by adding and/or changing some rules on simplex in order to shorten the track; even embedding it into a diameterorbit. The diameter of $P$ is the maximal distance in between arbitrary two vertices on $P$, while the distance of two vertices is the number of edges of the shortest track connecting the two vertices. A diameter shows a lower limit of pivot-steps in the edgefollowing algorithms with respect to the related LP problem. Hirsch guesses that the diameter of $k$ facets-polytope in $X=R^{d}$ is $k-d$. When $d=n$, the LP problem has $m$ non-natural constraint facets with $n$ natural constraint facets, the LP polytope has $k=m+n$ facets. According to the conjecture, the diameter is $k-d=m$. Based on his conjecture, the lower limit of steps of pivots taken in an edge-following algorithm is as small as $m$, and he firmly denies the existence of super polynomial diameters: if it exists, then there is no simplex variant that can run in polynomial time. Disproving of the conjecture may be a serious challenge for edge-following algorithms.

A counter-example for Hirsch conjecture was announced in May 2010 by Santos [4] who presented his findings at the conference 100 Years in Seattle: the mathematics of Klee and Grünbaum. This information raises more questions on the edge-following algorithms, and the scientific community becomes more interested about the open problem: Are there strongly polynomial-time algorithms for the LP problems existing still? This paper will give a positive answer to the problem. The analysis is based on the cone-cutting approach, which will be introduced in Sect. 2.

Motivated by the analysis on Hirsch conjecture, we are interested on the shortest path in or on the dual feasible region $D$, starting from a given $D$-point and ending at the lowest point $\boldsymbol{y}^{*}$. An algorithm named Gradient Falling will be introduced in Sect. 4. The algorithm has traced out a shortest path in the dual feasible region $D$, the author guesses, it is a strongly polynomial-time's algorithm for linear programming.

Organization of the paper: the basic theory of cones and cone-cutting are outlined in Sect. 2, the propositions without proofs are cited from Zhang [5] and Wang [6]. The analyses for the Hirsch conjecture are given in Sect. 3; the algorithm GradientFalling is presented in Sect. 4; some discussions are left in Sect. 5. 


\section{Basis Cones and Cone-Cutting in Dual Space}

An algorithm on linear programming named Cone-Rotating had been presented by Zhang [5], which performs simplex pivot on rows (instead of column-treatments) and abandons the tableaus to reduce the spaces and calculations by some rules. The basic idea is viewing the feasible region as a polytope constrained by linear inequalities and treating the basis as a cone in order to construct his cone-rotating theory intuitively. Even though he treats cones based on facets only, he is the first person that initiates the concept of cone-cutting. The author is not an expert in LP, but involved this area from the research on fuzzy non-linear programming [7] and had a paper on LP submitted to the International Conference on LP in 2009, Hainan, China. At that time, the author thought that the concept of cone-cutting was a new idea initiated by him and there was no any relationship between cone-cutting with Simplex. Zhang showed his book pressed about 20 years ago and encouraged the author to admit such a view point: cone-cutting is an equivalent form of simplex's pivot. Pang [8], as another one of the most helpful scholars, showed his works on dual programming and passed over the same view point. Since that time, the author has recognized the perfect characteristics of simplex, and contributes the cone-cutting as an intuitive vision to the development of simplex variants. The basic theory of cone-cutting had been stated in the paper [6], in which, the LP problem takes the maximum form. We will outline the cone-cutting theory, taking the minimum form, in the section without proofs.

A non-empty subset $F$ in $Y=R^{m}$, is called an affine set if $P+t(Q-P) \in F$ for any $P, Q \in F, t \in R^{1}$. An affine set $F^{\prime}$ is called parallel in $F$ if there is $P \in F$ such that $P+Q \in F$ for any $Q \in F^{\prime} . F^{\prime}$ is called parallel with $F$ if $F$ parallels in $F^{\prime}$ and $F^{\prime}$ parallels in $F$. An affine set containing the origin point $O$ is called a subspace of $Y$. If a nonempty affine set in $Y$ is not a subspace of $Y$, then it must parallel with a determinate subspace $Y^{\prime}=R^{m^{\prime}}\left(0 \leq m^{\prime}<m\right)$, and the number $m^{\prime}$ is called dimension of the nonempty affine set. Let $\boldsymbol{y}=\left(y_{1}, \ldots, y_{m}\right)$ be a row vector and $\boldsymbol{a}=\left(a_{1}, \ldots, a_{m}\right)^{T}$ be a (column) vector in the paper. A (super) plane $(x)$ is an $m-1$ dimensional affine set constrained by an equation $\boldsymbol{y} \boldsymbol{a}=c$, where ' $\boldsymbol{a}$ ' is the coefficient vector of the plane $(x)$ and ' $c$ ' is a real number. A plane $(x)$ is called a facet if it directs its interior (exterior) area by the inequality $\boldsymbol{y} \boldsymbol{a} \geq c(\boldsymbol{y} \boldsymbol{a}<c)$. A point $P$ in $Y$ is called accepted (rejected) by the facet if $\boldsymbol{y} \boldsymbol{a} \geq c(\boldsymbol{y} \boldsymbol{a}<c)$. The interior area of facet $(x)$ is denoted as

$$
(x)_{\geq}=\{\boldsymbol{y} \mid \boldsymbol{y} \boldsymbol{a} \geq c\} .
$$

Let $\boldsymbol{x}$ and $\boldsymbol{b}$ be two (column) vectors in the paper. A programming (P) $\max \{\boldsymbol{c x} \mid A \boldsymbol{x} \leq \boldsymbol{b}\}$ can be represented by the simplex tableau

$$
\left(\begin{array}{ccccccc}
a_{11} & \ldots & a_{1 n} & 1 & \ldots & 0 & b_{1} \\
& \ldots & & \ldots & & & \\
a_{m 1} & \ldots & a_{m n} & 0 & \ldots & 1 & b_{m} \\
-c_{1} & \ldots & -c_{n} & 0 & \ldots & 0 & 0
\end{array}\right)
$$

The lowest row of the tableau was $\left(c_{1}, \ldots, c_{n}, 0, \ldots, 0\right)$. Since simplex's pivot shows the dual basic solution in the reduced costs row but with a signal-difference, the 
public usage of simplex tableau puts $\left(-c_{1}, \ldots,-c_{n}, 0, \ldots, 0\right)$ as the reduced costs in order to show the solution positively. Neglecting a signal-difference in between them, the simplex tableau represents the coefficient matrix of dual programming (D) $\min \{\boldsymbol{y} \boldsymbol{b} \mid \boldsymbol{y} A \geq \boldsymbol{c}\}$. Column ${ }_{j}$ of $\left(T_{0}\right)$ corresponds to a non-natural or natural dual constraint planes in dual space $Y=R^{m}$ :

$$
\left(x_{j}\right): y A_{j}=c_{j} \quad(1 \leq j \leq n) ; \quad\left(x_{n+i}\right): y_{i}=0 \quad(1<i \leq m) .
$$

The cone-cutting theory gives the simplex tableau following new explanations:

\subsection{Meanings of the Original Simplex Tableau $\left(T_{0}\right)$ and Its Basis Cone}

Facing a tableau $(T)=(T)_{(m+1) \times(n+m+1)}$, denote the sub-matrix of tableau $(T)$ occupying rows from 1 to $m$ and columns from 1 to $n+m$ by $T$ and the sub-matrix occupying rows from 1 to $m$ and columns from 1 to $n+m+1$ by $T^{+}$. For any sub-matrix $S$ of $T^{+}$, the sub-matrix $\underline{S}$ stands for the augmented matrix of $S$ toward the lowest row of $(T)$. So that, $\underline{T}$ is the sub-matrix occupying rows from 1 to $m+1$ and columns from 1 to $n+m$, and $T^{+}$is the whole matrix of tableau $(T)$.

There is a square matrix $B_{0}=I$ occupying columns $n+1$ to $n+m$ of $\left(T_{0}\right), \underline{B}_{0}$ is called the basis matrix of $\left(T_{0}\right)$, the columns of $\underline{B}_{0}$ describe the natural constraint planes $\left(x_{n+i}\right): y_{i}=0$ for $1 \leq i \leq m$, they form the coordinate cone $C_{0}$, called the basis cone of $\left(T_{0}\right)$.

Cone $C_{0}$ has a vertex $V_{0}=O$ with $m$ edges started from $O$ and directed by unitvectors $\left\{\boldsymbol{e}_{i}\right\}_{(i=1, \ldots, m)}$ along the $m$ axes. Since $O=(0, \ldots, 0)$ is the row $m+1$ of $\underline{B}_{o}$ and $\left\{\boldsymbol{e}_{i}\right\}_{(i=1, \ldots, m)}$ are the row-vectors of $B_{o}, \underline{B}_{o}$ represents the edges of $C_{0}$ by its rows. The following proposition is true:

Proposition 2.1 The basis matrix $\underline{B}_{0}$ in $\left(T_{0}\right)$ describes the coordinate cone $C_{0}$ in $Y: C_{0}$ takes columns of $\underline{B}_{0}$ as the coefficient vectors of its facets and takes rows of $B_{0}$ as its edge-directions.

\subsection{Meanings of Successive Simplex Tableaus $(T)$ and Their Basis Cones}

Given a tableau $(T)$ obtained by pivots successively started from $\left(T_{0}\right)$, denote the index-set of basis variables in $(T)$ by $\operatorname{BASE}(T)=\{[1], \ldots,[m]\}$, where $[k]$ stands for the index of $k$ th basis variable. The sub-matrix of $(T)$ occupying columns $[1], \ldots,[m]$ is always a unit-matrix $I$; while the sub-matrix $B$ of $T_{0}$ occupying columns $[1], \ldots,[m]$ is called the $(T)$-basis square in $\left(T_{0}\right)$. It is obvious that $\operatorname{INDEX}(B)=\operatorname{BASE}(T)$. We call $\underline{B} .=\underline{B} \cdot(m+1) \times m$ the $(T)$-basis matrix in $\left(T_{0}\right)$, where $\underline{B}$. is the augmented matrix of $B$ but taking $\left(c_{[1]}, \ldots, c_{[m]}\right)$ as its row $\operatorname{ros}_{m+1}$. i.e., the basis matrix $\underline{B}$. equals to $\underline{B}$ except replacing $-c_{[j]}$ back to $c_{[j]}$ for $j=1, \ldots, m$.

Rewrite $\boldsymbol{c}=\left(c_{1}, \ldots, c_{n} ; 0, \ldots, 0\right)$, and denote $\boldsymbol{c}_{B}=\left(c_{[1]}, \ldots, c_{[m]}\right)$. Since $r(B)=$ $m$, the equation $\boldsymbol{y} B=\boldsymbol{c}_{B}$ offers a determinate solution in $Y$ :

$$
V=c_{B} B^{-1}
$$


We call coordinate transformation $\boldsymbol{y}^{\prime}=(\boldsymbol{y}-V) B$ the $B$-transformation on $Y$, and it has the inversed transformation $\boldsymbol{y}=\boldsymbol{y}^{\prime} B^{-1}+V$. By means of the $B^{-1}$-transformation, the plane-equations described by columns in $\left(T_{0}\right)$ are transferred to

$$
\left(x_{j}\right): \boldsymbol{y}^{\prime} B^{-1} A_{j}=\boldsymbol{c}_{B} B^{-1} A_{j}-c_{j} \quad(j \leq n) ; \quad\left(x_{n+j}\right): \boldsymbol{y}^{\prime} B_{j}^{-1}=\boldsymbol{c}_{B} B_{j}^{-1}(1<j \leq m) .
$$

They form exactly the simplex tableau for basis $B$ :

$$
\left(\begin{array}{ccc}
B^{-1} A & D=B^{-1} & B^{-1} b \\
c_{B} B^{-1} A-c_{A} & c_{B} B^{-1} & c_{B} B^{-1} b
\end{array}\right)
$$

where the sub-matrix $\underline{D}$ having $\operatorname{INDEX}(\underline{D})=\{n+1, \ldots, n+m\}$ is called the feature matrix of $(T)$. When $(T)=\left(T_{0}\right)$, we have that $\underline{D}=\underline{B}_{0}$; but $\underline{D}$ is different with the basis matrix $\underline{B}$. in tableau $(T)$ when $(T) \neq\left(T_{0}\right)$. Denote

$$
c_{j}^{\wedge}=c_{B} B^{-1} A_{j}-c_{j}(j \leq n) ; c_{j}^{\wedge}=c_{B} B_{j}^{-1}(n+1<j \leq n+m) .
$$

$\boldsymbol{c}^{\wedge}=\left(c_{1}^{\wedge}, \ldots, c_{n+m}^{\wedge}\right)$ is the reduced cost vector.

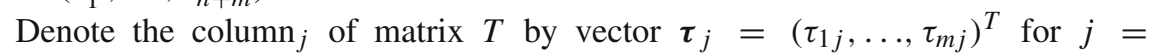
$1, \ldots, n+m$, the elements of matrix $T$ are unified. Denote column $n_{n+m+1}$ of $T^{+}$ by $\sigma=\left(\sigma_{1}, \ldots, \sigma_{m}\right)^{T}$, the whole matrix $\underline{T}^{+}$can be written as

$$
\underline{T}^{+}=\left(\begin{array}{cccc}
\tau_{1} & \ldots & \tau_{n+m} & \sigma \\
c_{1}^{\wedge} & \ldots & c_{n+m}^{\wedge} & z
\end{array}\right)
$$

where $z=c_{B} B^{-1} \boldsymbol{b}$ is the height of the dual basic solution, and for $i=1, \ldots, m$, and where

$$
\begin{aligned}
\tau_{i j}=\left(B^{-1} A\right)_{i j} & =(D A)_{i j}(1 \leq j \leq n) ; \tau_{i j}=d_{i j}(n+1<j \leq n+m) \\
\sigma_{i} & =(D \boldsymbol{b})_{i}=\boldsymbol{d}_{i} \boldsymbol{b}
\end{aligned}
$$

Since the reduced costs under the feature matrix $D$ is the vector $\boldsymbol{c}_{D}^{\wedge}=$ $\left(c_{n+1}^{\wedge}, \ldots, c_{n+m}^{\wedge}\right)$, according to (2.3), the solution of equation $\boldsymbol{y} B=\boldsymbol{c}_{B}$ can be written as:

$$
V=\left(c_{n+1}^{\wedge}, \ldots, c_{n+m}^{\wedge}\right)
$$

The constraint planes can be uniformly rewritten as

$$
\left(x_{j}\right): \boldsymbol{y}^{\prime} \boldsymbol{\tau}_{j}=-\boldsymbol{c}_{j}^{\wedge} \quad(j=1, \ldots, n+m) .
$$

Remember that the tableau $(T)$ is obtained from $\left(T_{0}\right)$, denote the columns of $\left(T_{0}\right)$ as $\boldsymbol{\tau}_{j}^{0}(j=1, \ldots, n+m)$. The formula (2.6) can be written as

$$
\tau_{i j}=\boldsymbol{d}_{i} \boldsymbol{\tau}_{j}^{0} \quad(j=1, \ldots, n+m) ; \quad \sigma_{i}=\boldsymbol{d}_{i} \boldsymbol{b} \quad(i=1, \ldots, m) .
$$


Definition 2.1 Let $\underline{B}$. be the $(T)$-basis matrix in $\left(T_{0}\right)$. The columns of $\underline{B}$. form a (facets) cone $C=C\left(\left(x_{j}\right) \mid j \in \underline{B}\right.$.) defined by

$$
C=\left\{\boldsymbol{y} \in Y \mid \boldsymbol{y} B \geq \boldsymbol{c}_{B}\right\}
$$

$C$ is called the basis cone of $(T)$, while $\underline{B}$. is called the facet matrix of $C$. Each plane $\left(x_{j}\right)$ with $j \in \operatorname{INDEX}(B)$ is called a $C$-facet. The set of indices of $C$-facets is denoted as $\operatorname{FACE}(C)$. We have that $\operatorname{FACE}(C)=\operatorname{BASE}(T)$.

The solution $V=\left(c_{n+1}^{\wedge}, \ldots, c_{n+m}^{\wedge}\right)$ of the equation $\boldsymbol{y} B=\boldsymbol{c}_{B}$ is called the vertex of $C$. For each $i \in\{1, \ldots, m\}$, there is a ray $E_{i}$, starting from $V$ and directing the interior of $\left(x_{i}\right)$ by a row vector $\boldsymbol{d}_{i}$, which lays on the line $L_{i}$ intersected by all $C$-facets except $\left(x_{i}\right) . E_{i}, L_{i}$ and $\boldsymbol{d}_{i}$ are called the edge, edge-line and edge-direction of $C$ respectively.

A cone can be also defined by edges or edge-directions:

Definition 2.1' Given a point $V$ and $m$ vectors with rank $r\left(\boldsymbol{d}_{1}, \ldots, \boldsymbol{d}_{m}\right)=m$, the (edge) cone $C=C\left(V ; \boldsymbol{d}_{1}, \ldots, \boldsymbol{d}_{m}\right)$ is a polytope formed by

$$
C=\left\{\boldsymbol{y} \mid \exists \boldsymbol{\xi}=\left(\xi_{1}, \ldots, \xi_{m}\right) \geq \mathbf{0} ; \boldsymbol{y}=V+\xi_{1} \boldsymbol{d}_{1}+\ldots+\xi_{m} \boldsymbol{d}_{m}\right\}
$$

The matrix $\left(\boldsymbol{d}_{1}, \ldots, \boldsymbol{d}_{m}, V\right)^{T}$ is called the edge matrix of $C$.

Each edge-direction $\boldsymbol{d}_{i}$ is unique up to a positive factor $\lambda_{i}$ independently. To get back to the feature square $D$, we need to determine factors $\lambda_{1}, \ldots, \lambda_{m}$ such that $\left(\lambda_{1} \boldsymbol{d}_{1}, \ldots, \lambda_{m} \boldsymbol{d}_{m}\right)^{T}=D$.

The two definitions are equivalent. Given a cone defined by facets $\left\{\left(x_{[j]}\right)\right\}_{(j=1, \ldots, m)}$ with rank $r=m$, there uniquely corresponds to a cone $C=C\left(V ; \boldsymbol{d}_{1}, \ldots, \boldsymbol{d}_{m}\right)$, where $V$ is the intersection of the given $C$-facets, while $\boldsymbol{d}_{i}$ lays on the line intersected by all $C$-facets except $\left(x_{[i]}\right)$ and directs to the accepted area of $\left(x_{[i]}\right)$; Inversely, given a cone defined by vertex $V$ and edge-directions $\mathbf{d}_{1}, \ldots, \boldsymbol{d}_{m}$, with $\operatorname{rank} r\left(\boldsymbol{d}_{1}, \ldots, \boldsymbol{d}_{m}\right)^{T}=m$, there corresponds a cone $C=C\left[\left(x_{[1]}\right), \ldots,\left(x_{[m]}\right)\right]$ : For each $i$, the facet $\left(x_{[i]}\right)$ : $\boldsymbol{y} \boldsymbol{\tau}_{[i]}=-c_{[i]}^{\wedge}$ contains all edges except $E_{i}$.

There are equations in between coefficient vectors $\boldsymbol{\tau}_{i}$ and edge-directions $\boldsymbol{d}_{j}$ :

$$
\boldsymbol{d}_{j} \boldsymbol{\tau}_{i}=0 \quad(j \neq i) ; \quad \boldsymbol{d}_{i} \boldsymbol{\tau}_{i}>0 . \quad(i \in \mathrm{FACE}(C))
$$

For each $i \in \operatorname{FACE}(C)$, the plane $\left(x_{[i]}\right)$ and the ray $E_{i}$ form an opposite couple.

When $m=n$ and when the basis $B$ runs to $B=A$, we have that $D=B^{-1}=A^{-1}$. It means that $A^{-1}$ is the edge matrix of cone $C$. We can define the inverse cone $C^{-1}$ of $C$, which takes all coefficient vectors of $C$ as its edge-directions. Then the matrix $A$ is the edge matrix of $C^{-1}$. Since $\left(A^{-1}\right)^{-1}=A$, we have that

$$
\left(C^{-1}\right)^{-1}=C \text {. }
$$

The facet-matrix and the edge-matrix of a cone are inverse each other. However, the most important theoretical meaning of cone-cutting is: The concept of inverse cone 
is the geometrical representation of inverse matrix. In this paper, we do not elaborate further on the inverse cone and its properties.

We can summarize the ideas mentioned above into the following proposition without proofs:

Proposition 2.2 Any tableau $(T)$ determines its basis cone $C: F A C E(C)=B A S E(T)$. $C$ takes columns of $(T)$-basis matrix $\underline{B}$. in $\left(T_{o}\right)$ as the coefficient vectors of its facets (i.e., $\underline{B}$. is its facet matrix) and takes rows of feature matrix $\underline{D}$ of $(T)$ as its edgedirections (i.e., $\underline{D}$ is its edge matrix).

\section{Points in detail:}

1 The facets of $C$ are given by the (T)-basis matrix $B$. in $\left(T_{0}\right)$.

2 The edges matrix of $C$ is given by $\underline{D}$ in tableau $(T)$, the $i$ th row of $D$ is the edgedirection $\boldsymbol{d}_{i}$ of $C$, the vertex $V=\left(c_{n+1}^{\wedge}, \ldots, c_{n+m}^{\wedge}\right)$ is shown in the lowest row of D.

3 For any $j, c_{j}^{\wedge}$ is a checking value: $\left(x_{j}\right)$ passes through $V$ if $c_{j}^{\wedge}=0 ;\left(x_{j}\right)$ accepts but does not pass through $V$ if $c_{j}^{\wedge}>0 ;\left(x_{j}\right)$ rejects $V$ if $c_{j}^{\wedge}<0$.

4 The dual objective vector $\sigma=D \boldsymbol{b}$ in $(T)$ is the dual objective vector under Btransformation, which can be used to check the tendency of edge-directions: $\boldsymbol{d}_{i}$ directs upward (downward) if $\sigma_{i} \geq 0\left(\sigma_{i}<0\right)$; $C$ is called stable if $\boldsymbol{\sigma} \geq \mathbf{0}$.

5 The elements $\tau_{i j}$ in $T$ can be used to check the reality of intersection $Q_{i j}$ : For a stable cone $C$ (i.e., $\boldsymbol{\sigma} \geq \mathbf{0}$ in $(T)), Q_{i j}$ is real if $c_{j}^{\wedge} \tau_{i j} \leq 0$ and $\tau_{i j} \neq 0$, is fictitious if $c_{j}^{\wedge} \tau_{i j}>0$, is none if $\tau_{i j}=0$.

\subsection{Cone-Cutting, the Description of Pivot}

Taking a pivot on tableau $(T)$, we can obtain a new tableau $\left(T^{\prime}\right)$. The basis of tableau is renewed as follows:

$$
\operatorname{BASE}\left(T^{\prime}\right)=\left(j \rightarrow \operatorname{BASE}(T) \rightarrow j^{\prime}\right) .
$$

It is called an index-alternation on basis. The indices $j$ and $j^{\prime}$ are called the entering and leaving indices respectively. A pivot is an index-alternation. An index-alternation may not be a simplex pivot, it is called a simplex pivot if $j^{\prime}=[i(j)]$ obeys $(2.10)$.

If $\boldsymbol{\sigma} \geq \mathbf{0}$, then the vertex $V$ of $C$ is the dual optimal point $V^{*}$ whenever it is accepted by all dual constraints. Otherwise, there must be a plane $\left(x_{j}\right)$ cutting off $V$ and comes the concept of cone-cutting. We call $\left(x_{j}\right)$ a cutter of $C$ if $c_{j}^{\wedge}<0$ in (T) (i.e., $\left(x_{j}\right)$ rejects the vertex $V$ of $C$ ).

Given a stable cone $C$, for $i=1$ to $m$, if $\tau_{i j}=\boldsymbol{d}_{i} \boldsymbol{\tau}_{j} \neq 0$, then calculate the intersection of $\left(x_{j}\right)$ on $E_{i}$ : Write $E_{i}$ in parameter form $\boldsymbol{y}=V+t \boldsymbol{d}_{i}$, substitute it into the equation of $\left(x_{j}\right): \boldsymbol{y} \boldsymbol{\tau}_{j}=-c_{j}^{\wedge}$, and get $\left(V+t \boldsymbol{d}_{i}\right) \boldsymbol{\tau}_{j}=-c_{j}^{\wedge}$. Taking the solution of the parameter $t$, the intersection is obtained:

$$
Q_{i j}:=V+t_{i j} \boldsymbol{d}_{i} \quad\left(t_{i j}=-c_{j}^{\wedge} / \tau_{i j}\right)
$$

The intersection $Q_{i j}$ is called real if $t_{i j} \geq 0$; it is called fictitious if $t_{i j}<0$. 
From (2.9), we get the relative height of intersection point $Q_{i j}$ with respect to vertex $V$ of the current cone, stable or unstable, as follows:

$$
\left(Q_{i j}-V\right) \boldsymbol{b}=t_{i j} \boldsymbol{d}_{i} \boldsymbol{b}=-\left(c_{j}^{\wedge} / \tau_{i j}\right) \sigma_{i}
$$

Let $\boldsymbol{\xi}:=\underline{D} \boldsymbol{\tau}_{j}$, which is a column vector with $m+1$ component, we can get

$$
t_{i j}=\left(c_{j}-\xi_{m+1}\right) / \xi_{i}
$$

If all $t_{i j}<0$, then $\left(x_{j}\right)$ fictitiously intersects all edges of $C$. In this case, the result of $C$ cut by $\left(x_{j}\right)$ is an empty set; otherwise, set

$$
i(j)=\operatorname{argmin}_{i}\left\{Q_{i j} \boldsymbol{b} \mid t_{i j}>0\right\}
$$

$Q_{i(j), j}$ is the lowest real intersection of $\left(x_{j}\right)$ on $C$-edges, which is called the cutpoint of $\left(x_{j}\right), E_{i(j)}$ is the edge of $C$ carrying the cut-point, which is called the main edge.

Procedure ConeCutting: Cutting a stable cone $C$ by a cutter $\left(x_{j}\right)$. If $\tau_{i j}<0$ for all $i$, then stop and output: dual infeasible! Else, a new edge cone $C^{\prime}=C^{\prime}\left(V^{\prime} ; \boldsymbol{d}_{1}^{\prime}, \ldots, \boldsymbol{d}_{m}^{\prime}\right)$ is generated as follows:

D1. The new vertex is the cut-point of $\left(x_{j}\right)$ on $C: V^{\prime}=Q_{i(j), j}$;

D2. The edge-direction on main edge does not change: $\boldsymbol{d}_{i(j)}^{\prime}=\boldsymbol{d}_{i(j)}$;

D3. If $\tau_{i j}=0$ ( $E_{i}$ parallels in $\left.\left(x_{j}\right)\right)$, then edge-direction does not change: $\boldsymbol{d}_{i}^{\prime}=\boldsymbol{d}_{i}$;

D4. If $\tau_{i j}>0\left(\mathrm{Q}_{i j}\right.$ is real $)$, then $\boldsymbol{d}_{i}^{\prime}=Q_{i j}-Q_{i(j) j}=\boldsymbol{d}_{i} / \tau_{i j}-\boldsymbol{d}_{i(j)} / \tau_{i(j) j}$;

D5. If $\tau_{i j}<0$ ( $Q_{i j}$ is fictitious), then $\boldsymbol{d}_{i}^{\prime}=Q_{i(j) j}-Q_{i j}=\boldsymbol{d}_{i(j)} / \tau_{i(j) j}-\boldsymbol{d}_{i} / \tau_{i j}$.

Denote the new cone as $C^{\prime}=C \backslash\left(x_{j}\right)$, the procedure to form the new cone is called a cone-cutting; $(i(j), j)$ is called the eye of cone-cutting; and the facet $\left(x_{[i(j)]}\right)$ is called the expelled facet.

Note. The symbol " $\backslash$ " used here is not the difference-operation in set theory. However, the set $C^{\prime \prime}=C \cap\left(\left(x_{j}\right)_{<}\right)^{c}=C \backslash\left(x_{j}\right)_{<}$and $C^{\prime}=C \backslash\left(x_{j}\right)$ are coincide in a neighborhood of the vertex $V$ of $C$, where, the symbol " $\backslash$ " in $C$ " is the difference-operation in set theory.

To perform the procedure, we can have the following sub-algorithm:

\section{Sub-algorithm ConeCutting}

Given the edge matrix $\underline{D}$ of cone $C$ and the coefficient vector $\tau_{j}$ (replacing $c_{j}^{\wedge}$ by $\left.-c_{j}^{\wedge}\right)$ of a cutter $\left(x_{j}\right)$

1. Compute $\xi:=\underline{D} \tau_{j} ;(\xi$ is a column vector with $m+1$ components)

For $i=1$ to $m, t_{i}:=\left(c_{j}-\xi_{m+1}\right) / \xi_{i}$ when $\xi_{i} \neq 0 ; t_{i}:=*$ when $\xi_{i}=0$;

2. For $i=1, \ldots, m$, if $t_{i}=*$, then put the symbol $*$ on the right side of row $i$; else calculate $Q_{i j}:=V+t_{i} \boldsymbol{d}_{i}$, and put symbol - on the right side of row $i$ when $t_{i}<0$, (the intersection is fictitious);

3. If there are signs - or * appearing on the right side of all rows of $C$, then stop with "Dual infeasible!"; Else, select $i=i(j)$ according to (2.10'), i.e., select the 
lowest real intersection as new vertex: $V^{\prime}=Q_{i(j) j}$ and replace the $\operatorname{row}_{m+1}$ by the coordinate of $V^{\prime}$;

4. For $i=1, \ldots, m, i \neq i(j)$ and $t_{i} \neq *$, replace row $_{i}$ by the coordinate of $Q_{i j}$; and then minus row $_{m+1}$; and then multiplied by -1 if there is symbol - on the right side;

The resulted matrix is the new edge matrix $\underline{D}^{\prime}$ of new cone $C^{\prime}=C \backslash\left(x_{j}\right)$.

Cone-cutting is an intuitive representation of simplex's pivot. The key point is that formula (2.10') is equivalent to the following formula

$$
i(j)=\operatorname{argmin}_{i}\left\{\sigma_{i} / \tau_{i j} \mid \tau_{i j}>0\right\} \quad\left(c_{j}^{\wedge}<0\right) .
$$

Indeed, the intersection of $\left(x_{j}\right)$ on $E_{i}$ is $Q_{i j}=V+t_{i j} \boldsymbol{d}_{i}=V-\left(c_{j}^{\wedge} / \tau_{i j}\right) \boldsymbol{d}_{i}$ and the height of the intersection is $Q_{i j} \boldsymbol{b}=V \boldsymbol{b}-\left(c_{j}^{\wedge} / \tau_{i j}\right) \boldsymbol{d}_{i} \boldsymbol{b}$. Since $\boldsymbol{d}_{i} \boldsymbol{b}=\sigma_{i}$, we have that

$$
Q_{i j} \boldsymbol{b}=V \boldsymbol{b}-c_{j}^{\wedge} \sigma_{i} / \tau_{i j}
$$

Since $\left(x_{j}\right)$ is a cutter of cone $C$, we have $c_{j}^{\wedge}<0$ and hence $-c_{j}^{\wedge}>0$. In this case, $Q_{i * j}$ is the lowest real intersection if and only if $\sigma_{i *} / \tau_{i * j}$ takes the minimum of $\sigma_{i} / \tau_{i j}$ among all real intersections. According to Point 5 of Proposition 2.2, a stable cone has real intersection if and only if $\tau_{i j}>0$. Therefore, we have that

$$
\operatorname{argmin}_{i}\left\{Q_{i j} \boldsymbol{b} \mid t_{i j}>0\right\}=\operatorname{argmin}_{i}\left\{\sigma_{i} / \tau_{i j} \mid \tau_{i j}>0\right\} .
$$

It means that the rule (2.10) is the same as rule (2.10'). This is the core of the equivalence between cone-cutting and simplex's pivot.

Definition 2.2 A cone $C$ is called the cap of dual feasible region $D$ at a vertex $V \in D$ if $V$ is a vertex of $C$ and $C$ is coincide with $D$ in a neighborhood of $V$.

Related results are summed into the following proposition without proof.

Proposition 2.3 If the cutter $\left(x_{j}\right)$ does not cut a cone $C$ that results an empty set, then the new cone taken by cone-cutting $C^{\prime}=C \backslash\left(x_{j}\right)$ is the cap of $C \cap\left(x_{j}\right)_{\geq}$at $V^{\prime}$. The procedure ConeCutting is equivalent to the pivot of simplex:

1. Cone-cutting $C^{\prime}=C \backslash\left(x_{j}\right)$ realizes the index-alternation $B A S E\left(T_{k}\right)=(j \rightarrow$ $\left.B A S E\left(T_{k-1}\right) \rightarrow[i(j)]\right)$. The cutter $\left(x_{j}\right)$ and the expelled facet $\left(x_{[i(j)]}\right)$ represent the entering and leaving variables of pivot respectively. In this sense.

2. Cone-cutting keeps a stable cone to a new stable cone;

3. The cutter $\left(x_{j}\right)$ occupies the place of expelled facet $\left(x_{[i(j)]}\right)$ in basis, the occupier and the expelled are opposite to the main edge $E_{i(j)}$; While the expelled facet becomes an accepter of the new vertex of basis cone, i.e., $c_{[i(j)]}^{\wedge}>0$ in $\left(T^{\prime}\right)$; The relation $\sigma_{i(j) j} \neq 0$ always holds.

4. The vertex $V$ becomes the dual optimal point $V^{*}$ whenever $V$ is dual feasible. The new cut-point is always not lower than current vertex $V$, and the current vertex $V$ goes to $V^{*}$ upwards monotonically in the cone-cuttings' optimal searching. 
Example 2.1 Problem (P): $\max z=2 x_{1}-x_{2}$

$$
\begin{array}{cl}
\text { Sub. } & x_{1}-x_{2} \leq 1 \\
& x_{1}+x_{2} \leq 2 \\
& x_{1}, x_{2} \geq 0
\end{array}
$$

Dual problem (D): $\min w=y_{1}+2 y_{2}$

$$
\begin{array}{ccc}
\text { Sub. } & \left(x_{1}\right): & y_{1}+y_{2} \geq 2, \\
& \left(x_{2}\right): & -y_{1}+y_{2} \geq-1, \\
& \left(x_{3}\right): & y_{1} \geq 0, \\
& \left(x_{4}\right): & y_{2} \geq 0 .
\end{array}
$$

The dual objective vector is given by $\boldsymbol{b}=(1,2)^{T}$ and satisfies that $\boldsymbol{b} \geq \mathbf{0}$. As mentioned before, the vertex $V=(0,0)$ of the coordinate cone $C_{0}$ is dual optimal point if it is accepted by all constraint planes. Since $V$ is rejected by $\left(x_{1}\right), V$ is not optimal. We need to performing algorithm ConeCutting $C^{\prime}=C_{0} \backslash\left(x_{1}\right)$ as follows:

The edge matrix $\underline{D}_{0}$ of coordinate cone $C_{0}$ is written as follows:

$$
\begin{array}{ll}
1 & 0 \\
0 & 1 \\
0 & 0
\end{array}
$$

$V=(0,0) ; \quad \boldsymbol{d}_{1}=(1,0), \boldsymbol{d}_{2}=(0,1) ; \quad \boldsymbol{\sigma}_{1}=(1,1)^{T}, c_{1}=2$

According to stage 1 , calculate

$$
\begin{aligned}
& \xi=\underline{D}^{o} \sigma_{1}=(1,1,0)^{T} \\
& t_{1}=\left(c_{1}-\xi_{3}\right) / \xi_{1}=2, \quad t_{2}=\left(c_{1}-\xi_{3}\right) / \xi_{2}=2, \quad t=(2,2)^{T} .
\end{aligned}
$$

Since $t$ has two positive components, the facet $\left(x_{1}\right)$ has two real intersections: $Q_{11}=V+t_{1} \boldsymbol{d}_{1}=(2,0), Q_{21}=V+t_{2} \boldsymbol{d}_{2}=(0,2)$. Since $Q_{11} \boldsymbol{b}=2<Q_{21} \boldsymbol{b}=$ 4, $Q_{11}$ is the lowest real intersection and $V_{1}=Q_{11}=(2,0)$.

The main edge-line is $L_{1}$, and $V_{1}=Q_{11}$. Replace the row $_{3}$ by the coordinate of $Q_{11}$ :

$$
\begin{array}{ll}
1 & 0 \\
0 & 1 \\
2 & 0
\end{array}
$$

Apart of the main row, replacing row 2 by the coordinate of $Q_{21}$, and subtracting row 3 yields:

$$
\begin{array}{cc}
1 & 0 \\
-2 & 2 \\
2 & 0
\end{array}
$$


Fig. 1 Cone-cutting

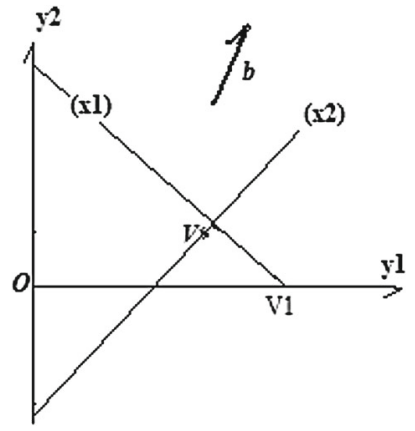

This is the edge matrix $\underline{D}_{1}$ of new cone $C_{1}=C_{0} \backslash\left(x_{1}\right)$. As shown in Fig. 1, new vertex $V_{1}=(2,0)$. New edge $E_{1}^{\prime}$ of $C_{1}$ has common edge-line $L_{1}=O V_{1}$ with the older edge $E_{1}$ of $C_{0}$, which is the main line in the cone-cutting. The edge-direction of main line is invariant, $\boldsymbol{d}_{1}^{\prime}=\boldsymbol{d}_{1}=(1,0)$.

Since $V_{1} \sigma_{2}=(2,0)(-1,1)^{T}=-2<-1, V_{1}$ is rejected by $\left(x_{2}\right)$, so that $V_{1} \neq V^{*}$. Continue to perform ConeCutting $C_{2}=C_{1(x 2)}$.

$$
\xi:=\underline{D}_{1} \sigma_{2}=(-1,4,-2)^{T} \circ
$$

$\boldsymbol{t}:=(-1,1 / 4)^{T}$. Since $t_{1}=-1<0, Q_{12}$ is fictitious, $Q_{12}=V_{1}+t_{1} \boldsymbol{d}_{1}^{\prime}=(2,0)-$ $(1,0)=(1,0)$. Put the negative sign on the right side of row 1 ; Since $t_{2}=1 / 4>0, Q_{22}$ is the only one real intersection and $Q_{22}=V_{1}+t_{2} d_{2}^{\prime}=(2,0)+(-2,2) / 4=$ $(3 / 2,1 / 2)$, and then $V_{2}=Q_{22}$. The main edge is $E_{2}^{\prime}$. i.e., $i(2)=2$. Replace the row 3 by the coordinate of $Q_{22}$ :

$V_{2}:=Q_{22}=(3 / 2,1 / 2)$.

$$
\begin{array}{cc}
1 & 0 \\
-2 & 2 \\
3 / 2 & 1 / 2
\end{array}
$$

Apart the main row, row 1 has no symbol ${ }^{*}$, replace row 1 by the coordinate of $Q_{12}$ and minus row 3 .

$$
\begin{array}{ccc}
-1 / 2 & -1 / 2 & - \\
-2 & 2 & \\
3 / 2 & 1 / 2 &
\end{array}
$$

Since there is sign - on the right side of row $_{1}$, multiplies -1 on the row, and delete the negative sign:

$$
\begin{array}{cc}
1 / 2 & 1 / 2 \\
-2 & 2 \\
3 / 2 & 1 / 2
\end{array}
$$


This is the edge matrix $D_{2}$ of the new cone $C_{2}=C_{1} \backslash\left(x_{2}\right)$. Since the new vertex $V_{2}$ is accepted by all dual constraints, we get the dual optimal point $V^{*}=V_{2}=(3 / 2,1 / 2)$. i.e., $y_{1}^{*}=3 / 2, y_{2}^{*}=1 / 2$. The dual optimal value is $w=V^{*} \boldsymbol{b}=5 / 2$.

Comparing the example, we can do simplex pivots on the problem $(\mathrm{P})$ :

$$
\begin{array}{cccccc} 
& X_{1} & x_{2} & x_{3} & x_{4} & z \\
x_{3} & 1 & -1 & 1 & 0 & 1 \\
x_{4} & 1 & 1 & 0 & 1 & 2 \\
& -2 & 1 & 0 & 0 & 0
\end{array}
$$

Since $c_{1}^{\wedge}<0$, enter $j=1$ into basis. According to (2.10), $i(1)=1$. Do pivot at eye $(1,1)$ and get

$$
\begin{array}{cccccc} 
& X_{1} & x_{2} & x_{3} & x_{4} & Z \\
x_{3} & \mathbf{1} & -1 & 1 & 0 & 1 \\
x_{4} & 0 & 2 & -1 & 1 & 1 \\
& 0 & -1 & 2 & 0 & 2
\end{array}
$$

Compare with the algorithm ConeCutting, we find out that the edge matrix of $C_{1}$ is

$$
\begin{array}{cc}
1 & 0 \\
-2 & 2 \\
2 & 0
\end{array}
$$

The row 2 in $\underline{D}_{1}$ is $(-2,2)$, but in tableau $\left(T_{1}\right)$ it becomes $(-1,1)$. Since they are proportionate each other, they point to the same direction. The edge matrix of $C_{1}$ is consistent with the feature matrix of tableau $\left(T_{1}\right)$. i.e., the rows of $D$ are proportionate to the rows of $C^{\prime}$ respectively.

Since $c_{2}^{\wedge}=-1<0$ in $\left(T_{1}\right)$, take $j=2$ to enter basis, $i(2)=2$. Do pivot at eye $(2,2)$ and get

$$
\begin{array}{cccccc} 
& X_{1} & x_{2} & x_{3} & x_{4} & \boldsymbol{Z} \\
x_{3} & 1 & 0 & 1 / 2 & 1 / 2 & 3 / 2 \\
x_{4} & 0 & \mathbf{1} & -1 / 2 & 1 / 2 & 1 / 2 \\
& 0 & 0 & 3 / 2 & 1 / 2 & 5 / 2
\end{array}
$$

Compare with the algorithm ConeCutting, we find that the edge matrix of $C_{2}$ is

$$
\begin{array}{cc}
1 / 2 & 1 / 2 \\
-2 & 2 \\
3 / 2 & 1 / 2
\end{array}
$$

The matrix of $C_{2}$ is consistent with the feature matrix of tableau $\left(T^{*}\right)$.

The optimal solutions are $x^{*}=\left(x_{1}^{*}, x_{2}^{*}\right)=(3 / 2,1 / 2) ; V^{*}=\left(y_{1}^{*}, y_{2}^{*}\right)=$ $(3 / 2,1 / 2) ; z=w=5 / 2$. 
The complexity of the algorithm ConeCutting is $O(\mathrm{~nm})$, which is the same as the complexity of pivot. Simplex's tableau operation is an elegant procedure in mathematical theory and applications, the author does not mean to use cone-cutting to replace simplex, but provide an intuitive interpretation on the pivoting operation. The best way is combine cone-cutting into pivot. In the rest of this paper, we will mix terminologies and denotations in usage.

The intuition of cone-cutting helps us to get some important propositions. For examples, if a plane $(x)$ intersects all edges of a cone fictitiously and rejects the vertex of the cone $C$, then it rejects the whole cone. Since the dual feasible region $D$ is contained by $C,(x)$ rejects whole $D$. It means that $(x)$ cuts $D$ to be empty, and the LP Problem is dual infeasible. Let $(-x)$ be the inversed plane of $(x)$. It is obvious that $(x)$ cuts $D$ to be empty, if and only if $(-x)$ is delete-able. According to the Point 5 of Proposition 2.2, for a stable cone $C, Q_{i j}$ is real if $c_{j}^{\wedge} \tau_{i j} \leq 0$ and $\tau_{i j} \neq 0$, is fictitious if $c_{j}^{\wedge} \tau_{i j}>0$, is none if $\tau_{i j}=0$, we can get the proposition 2.5 and 2.4 successively:

Proposition 2.4 In current tableau $(T)$ with $\sigma \geq 0$, if there is an index $j \in J$ such that $c_{j}^{\wedge}>0$ and $\tau_{i j} \geq 0$ for all $i \in\{1, \ldots, m\}$, then the dual objective point $V^{*} \notin\left(x_{j}\right)$ and $\left(x_{j}\right)$ is delete-able.

Proposition 2.5 In current tableau $(T)$ with $\sigma \geq 0$, if there is an index $j \in J$ such that $c_{j}^{\wedge}<0$ and $\tau_{i j} \leq 0$ for all $i \in\{1, \ldots, m\}$, then the dual feasible region $D=\varnothing$, the LP problem is dual infeasible.

\section{Cone Renovation and Hirsch Conjecture}

In this section, we are going to analyze Hirsch conjecture by means of cone renovation

Definition 3.1 Giving a prime problem $(\mathrm{P}) \max \{z=\boldsymbol{c x} \mid A \boldsymbol{x} \leq \boldsymbol{b}, \boldsymbol{x} \geq \mathbf{0}\}$, following subset $\underline{P}$ in $\underline{X}=R^{n+m}$ is called a $L P$ polytope with respect to $(\mathrm{P})$ :

$$
\begin{aligned}
& \underline{P}=\left\{\underline{x}=\left(\boldsymbol{x} ; x_{n+1}, \ldots, x_{n+m}\right) \mid A_{i} x+x_{n+i} \leq b_{i},(1 \leq i \leq m)\right. \\
& \left.x_{i} \geq 0(1 \leq i \leq n+m)\right\} .
\end{aligned}
$$

$\underline{P}$ is called regular if there is an objective vector $c$ such that the optimal point $\underline{x}^{*}$ (with respect to $\boldsymbol{c})$ takes the longest distance of $\underline{P}$ from $\underline{\boldsymbol{x}}_{0}$, where $\underline{\boldsymbol{x}}_{0}=\left(0, \ldots, 0 ; b_{1}, \ldots b_{m}\right)$.

From the definition, we know that $\underline{P}$ is the prime feasible region for the standard simplex form of LP problem, which includes relaxed variables $x_{n+1}, \ldots, x_{n+m}$. Note that the convex set $\underline{P}$ is independent with the selection of prime objective vector $c$. Set $d=n+m$, let $\underline{P}$ be a regular LP polytope in $\underline{X}=R^{d}$. The number of facets of $\underline{P}$ is $m+(n+m)=d+m$. In this case, the Hirsch conjecture could be stated as follows: Hirsch conjecture. The diameter of an $m+d$ polytope in $R^{d}$ is $m$.

Where the diameter of $\underline{P}$ is the maximum of distances $d_{i j}$, the number of edges of the shortest path connecting vertices $V_{i}$ and $V_{j}$ on $\underline{P}$. The larger number of facets and the lower dimension yields longer diameter. He guesses the diameter is the difference between the number of facets and the dimensional number. The following conjecture is the simplest version of Hirsch conjecture: 
$\boldsymbol{m}$-step conjecture. (Klee and Walkup [9]) The diameter of $2 m$-facets polytope in $R^{m}$ is $m$.

With the understanding from the author, a slightly different statement could be presented on Terlaky assembling conjecture (See Terlaky [10]) as follows:

Prime assembling conjecture. Given a LP polytope $\underline{P}$ with tableau $\left(T_{0}\right)$, we can get optimal solution within $m$ steps of simplex-pivots (obeying (2.10)) provided the objective basis $B^{*}$ is known.

According to the cone-cutting theory, each simplex-pivot is a cone-cutting performed on the dual space. Hence the prime assembling conjecture is equivalent the following conjecture:

Dual assembling conjecture. Given a dual LP problem (D), we can get dual optimal point $V^{*}$ in $Y=R^{m}$ within $m$ steps of cone-cutting provided that all objective facets of $C^{*}$ are known.

Since the vertex of a cone is cut off by a cone-cutting, the track of vertices in dual space is outside the dual feasible region $D$. The series of cone-cuttings mentioned in the conjecture is not an edge-following but an exterior point method in the dual space, which corresponds to an edge-following algorithm for proving the prime assembling conjecture.

Proposition 3.1 For a regular LP polytope $\underline{P}$, Hirsch conjecture is true if and only if the prime assembling conjecture is true.

Proof Suppose that Hirsch conjecture is true for a LP polytope $\underline{P}$, we are going to prove that the assembling conjecture is true. i.e., if the optimal basis $B^{*}$ is known, then there is a series of simplex pivots such that the optimal prime solution $\underline{x}^{*}$ can be obtained within $m$ steps. Since Hirsch conjecture is true for $\underline{P}$, the diameter of $\underline{P}$ is $m$. It means that the distance between any two vertices of $\underline{P}$ have a distance is smaller than or equal to $m$. Let $S$ be the shortest path connecting vertices: $\left(0, \ldots, 0 ; b_{1}, \ldots, b_{m}\right)=$ $\underline{x}^{0}, \underline{\boldsymbol{x}}^{1}, \ldots, \underline{x}^{s-1}$ and $\underline{\boldsymbol{x}}^{s}=\underline{\boldsymbol{x}}^{*}$ successively, the distance in between $\underline{\boldsymbol{x}}^{0}$ and $\underline{x}^{*}$ is $s \leq m$. For $k=0, \ldots, s, \underline{x}^{k}$ is a prime basic solution with respect to a basis $B^{k}$, which satisfying $T_{0} \underline{x}^{k}=\boldsymbol{b}$, where $T_{0}$ is the coefficient matrix of original tableau $\left(T_{0}\right)$, Similarly, $\underline{x}^{k+1}$ is a prime basic solution with respect to a basis $B^{k+1}$, which satisfying $T_{0} \underline{\boldsymbol{x}}^{k+1}=\overline{\boldsymbol{b}}$. Since $\underline{\boldsymbol{x}}^{k} \underline{\boldsymbol{x}}^{k+1}$ is an edge of $\underline{P}$, the two groups of equations determining $\boldsymbol{x}^{k}$ and $\boldsymbol{x}^{k+1}$ must have $d-1$ common variables. So that, the two bases $B^{k}$ and $B^{k+1}$ have $m-1$ common columns, and then, there is a pivot transfer the prime basic solution from $\underline{\boldsymbol{x}}^{k}$ to $\underline{\boldsymbol{x}}^{k+1}$. Since $\boldsymbol{b} \geq \mathbf{0}$, a simplex pivot is taken. When $k=s$, we can get $\underline{\boldsymbol{x}}^{*}$ with $s \leq m$ steps of simplex pivots. The prime assembling conjecture is true for the LP polytope $\underline{P}$.

Conversely, suppose that the prime assembling conjecture is true for $\underline{P}$. Since $\underline{P}$ is convex, any vertex $\underline{x}$ of $\underline{P}$ could be the prime optimal point $\underline{x}^{*}$ provided that the objective vector $\boldsymbol{c}$ is selected upwards when we stand at $\underline{x}$ above $\underline{P}$. Since $\underline{P}$ is independent to the selection of prime objective vector $c$, it is reasonable to suppose that $\underline{\boldsymbol{x}}=\underline{\boldsymbol{x}}^{*}$ with respect to $\boldsymbol{c}$. Since the prime assembling conjecture is true for $\underline{P}$, and since the track of shortest path $S$ connecting $\underline{x}^{0}$ and $\underline{x}^{*}$ is from vertex to vertex on $\underline{P}$, the two point $\underline{x}^{0}$ and $\underline{x}^{*}$ have distance $s \leq m$. Since the vertex $\underline{x}$ is an arbitrary vertex of $\underline{P}$ and since the estimation of diameter of $\underline{P}$ can start from $\underline{x}^{0}$, the diameter of $\underline{P}$ is $m$, and Hirsch conjecture is true for $\underline{P}$. 
It is very easy to get an algorithm to get the optimal solution within $d(\leq m)$ steps of pivots provided that the objective indices are known. Unfortunately, the pivots performed in the algorithm are not limited to the simplex pivots. i.e., the leaving variable $i$ in eye $(i, j)$ may not be the cut-point index $i=i(j)$ defined by (2.10). Therefore, the vertex of current cone may not be a vertex of $\underline{P}$. The algorithm is not available for the proof of assembling conjecture. We are going to conditionally prove the dual assembling conjecture by means of cone-cuttings.

Dual assembling conjecture plainly represents $m$-step conjecture, while it is indeed a cone-renovation problem from cone-cutting's view point. i.e., it transfers the dual original cone $C_{0}$ to the dual objective cone $C^{*}$.

Definition 3.2 Let $C$ and $C^{\prime}$ be two stable cones with vertex $V^{\prime} \in C$, a procedure performing a series of simplex-pivots/cone-cuttings from $C$ to $C^{\prime}$, denoted as $C \rightarrow C^{\prime}$, is called a cone-renovation.

The dual assembling problem becomes: Can a cone-renovation $C^{o} \rightarrow C^{*}$ be realized within $m$ steps of cone-cutting? The key problem is: How to ensure that a red facet is not expelled by a red facet in each step of cone-cutting?

Definition 3.3 We call a cone-cutting has internal confliction if a red index expels out a red index.

If $C^{*}$ keeps cutting the current cone uniformly, then the cone-cuttings with internal confliction will be avoid.

Definition 3.4 We call that $C^{*}$ fully cuts a cone $C$ if all red non- $C$-facets cut off (i.e., reject) the vertex $V$ of $C$.

Definition 3.5 We call $C^{*}$ uniformly cuts $C$ if $C^{*}$ fully cuts $C$ and the cut-points of them are uniformly distributed on those $C$-edges opposite to non- $C^{*}$ facets.

If there are $c(0 \leq c<m)$ common facets of $C$ and $C^{*}$, then there are $m-c \quad C^{*}$ facets being not $C$-facets and there are $m-c \quad C$-facets being not $C^{*}$-facets. Note that a facet is black if it is not a $C^{*}$-facet, hence there are $m-c$ black $C$-facets, they are opposite to $m-c \quad C$-edges, which are called black edges of $C$. $C^{*}$ uniformly cuts $C$ if and only if each black edge of $C$ holds a cut-point, and different cut-points are cut by different $C^{*}$-facets. (i.e. each of $m-c$ non-common $C^{*}$-facets has a cut-point at each of the black edges).

Proposition 3.2 If $C^{*}$ uniformly cuts $C$, then take any non-common $C^{*}$-facet $\left(x_{j}\right)$ as a cutter, the cone-cutting $C \backslash\left(x_{j}\right)$ does not have internal confliction.

Proof According to Point 3 of Proposition 2.3, the cutter $\left(x_{j}\right)$ occupies the place of expelled facet $\left(x_{[i(j)]}\right)$ in basis, the occupier and the expelled are opposite to the same main edge $E_{i(j)}$. Since $C^{*}$ uniformly cuts $C$, the cut-points of all red non- $C$ facets are located on those $C$-edges opposite to non- $C^{*}$ facets. Therefore the expelled facets are non- $C^{*}$ facets, and then there is no red facet expelled out by non- $C$ red facets. Thus the cone-cutting $C \backslash\left(x_{j}\right)$ does not have internal confliction.

It is obvious that $C^{*}$ fully cuts $C$ if $C^{*}$ uniformly cuts $C$, and the inversed proposition is shown as follows: 

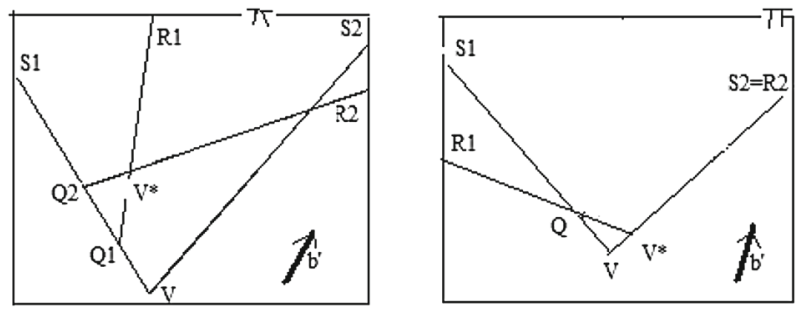

Fig. 2 a Picture for proof, part 1. b Picture for proof, part 2

Proposition 3.3 In the cone-renovation problem $C \rightarrow C^{*}, C^{*}$ uniformly cuts $C$ if $C^{*}$ fully cuts $C$.

Proof Firstly, we are going to prove that there is no more than one cut-points located on a same $C$-edge.

Suppose that there is a $C$-edge $E$ holding two cut-points $Q_{1}$ and $Q_{2}$. If $V^{*}$ is located at $E$, then $V^{*}$ is the intersection of a red non-C facet on $E$, denoted it as $(x)$, and then $C^{*}=C \backslash(x)$. We can get $C^{*}$ immediately, and the cone-renovation is realized; Otherwise, the optimal point $V^{*}$ is not located at $E$. Then a 2-dimensional plane $\pi$ passing through $E$ and $V^{*}$ can be determined. The intersections of $C$ and $C^{*}$ on $\pi$ are two angles $\angle V$ and $\angle V^{*}$ respectively. As shown in the Fig. 2(a), $\angle V=\angle S_{1} V S_{2}$ and $\angle V^{*}=\angle R_{1} V^{*} R_{2}$, where the ray $V S_{1}$ is the very edge $E$. The two cut-points $Q_{1}, Q_{2}$ are the intersections of $E$ with lines $V^{*} R_{1}, V^{*} R_{2}$ respectively. Let lines $V^{*} R_{1}, V^{*} R_{2}$ be the intersection of $\left(x_{1}\right)$ and $\left(x_{2}\right)$ on $\pi$, lines $V^{*} R_{1}$ and $V^{*} R_{2}$ inherit the respective directions of red non- $C$ facets $\left(x_{1}\right)$ and $\left(x_{2}\right)$ on $\pi$ too. Suppose that $Q_{1}$ is in between $V$ and $Q_{2}$. Since both facets $\left(x_{1}\right)$ and $\left(x_{2}\right)$ reject $V$, lines $V^{*} R_{1}$ and $V^{*} R_{2}$ reject $V$ too, and then, the common interior areas accepted by the two lines on the edge $V S_{1}$ is the ray $Q_{2} S_{1}$. Therefore, the angle formed by the two lines is the angle $\angle R_{1} V^{*} Q_{2}$. Since $C^{*}$ is stable, the ray $V^{*} R_{2}$ is upward with respect to $\boldsymbol{b}^{\prime}$, the projection of the dual objective vector $\boldsymbol{b}$ on $\pi$. Since $V^{*} Q_{2}$ is the inversed ray of ray $V^{*} R_{2}, V^{*} Q_{2}$ is downward with respect to $\boldsymbol{b}^{\prime}$. Then the angle $\angle V^{*}$ is not stable with respect to $\boldsymbol{b}^{\prime}$. But an intersected angle of a stable cone must be stable. This is a contradiction. Suppose that $Q_{2}$ is in between $V$ and $Q_{1}$, it presents a contradiction also. It means that the assumption is not true. Therefore, the first part of proposition is true.

Secondly, we are going to prove that the cut-points of non- $C$ red facets are located on the black $C$-edges, they are opposite to black facets.

Suppose that there is a red non- $C$ facet $\left(x_{1}\right)$ having a cut-point $Q$ that can not be located on a black edge, but on a $C$-edge $E$ opposite to a red non- $C$ facet $\left(x_{2}\right)$. Since $\left(x_{2}\right)$ is red, $V^{*} \in\left(x_{2}\right)$. Since $E$ is opposite to $\left(x_{2}\right), V^{*} \notin E$. Hence a 2-dimensional plane $\pi$ passing through $E$ and $V^{*}$ can be determined. The intersections of $C$ and $C^{*}$ on $\pi$ are two angles $\angle V$ and $\angle V^{*}$ respectively. As shown in the Fig. $2 \mathrm{~b}, \angle V=\angle S_{1} V S_{2}$ and $\angle V^{*}=\angle R_{1} V^{*} R_{2}$, where the ray $V S_{1}$ is the very edge $E$, line $V S_{2}=V^{*} R_{2}$ is the intersection of $\left(x_{2}\right)$ on $\pi$, and line $V^{*} R_{1}$ is the intersection of $\left(x_{1}\right)$ on $\pi$. Since $V^{*} \in\left(x_{2}\right), V^{*} \in V S_{2}$ too. Since $Q$ is the cut-point of $\left(x_{1}\right)$, it is the lowest point of $C \cap\left(x_{1}\right)_{\geq}$. Since the cut-point of $\left(x_{1}\right)$ can not be located on a black edge, $\left(x_{1}\right)$ has no intersection $Q^{\prime}$ on any black edges with same height as $Q$. Therefore, $Q$ is lower than 
any point in $C \cap\left(x_{1}\right)_{\geq}$. Since $V^{*} \in C \cap\left(x_{1}\right)_{\geq}, Q$ is lower than $V^{*}$. But, Since cone $C^{*}$ is stable, $\angle V^{*}$ is stable with respect to $\boldsymbol{b}^{\prime}$, the projection of dual objective vector $\boldsymbol{b}$ on $\pi$, i.e., the ray $V^{*} R_{1}$ is not downward with respect to $\boldsymbol{b}^{\prime}$. Since $Q$ is located on the ray $V^{*} R_{1}, Q$ is not lower than $V^{*}$. This is a contradiction. Suppose that $Q_{2}$ is in between $V$ and $Q_{1}$, it presents a contradiction also. It means that the assumption is not true. Hence the second part of proposition is true.

Definition 3.6 Let $C_{k}=C_{k-1} \backslash\left(x_{(k)}\right)$ be a cone-cutting (can be performed by pivot on tableaus $\left(T_{k-1}\right)$, and $C_{k-1}$ is the basis cone of $\left(T_{k-1}\right)$ ) for $k=1,2, \ldots$ We say the series of cone-cuttings obeys the lowest cut principle if the cutters $\left(x_{(k)}\right)$ are selected according to:

$$
(k)=\operatorname{argmax}_{j}\left\{c_{j}^{\wedge} \sigma_{i(j)} / \tau_{i(j), j} \text { in }\left(T_{k-1}\right) \mid j \notin \operatorname{FACE}\left(C_{k-1}\right)\right\} .
$$

Since the relative height of the cut-point of $\left(x_{j}\right)$ on cone $C_{k-1}$ is $-c_{j}^{\wedge} \sigma_{i(j)} / \tau_{i(j), j}$ in $\left(T_{k-1}\right)$, and according to $(3.1),\left(x_{(k)}\right)$ has the lowest cut-point on $C_{k-1}$ among all cutters. That is why we call (3.1) the lowest cut principle.

Proposition 3.4 If the cutter $\left(x_{(k)}\right)$ is selected by the lowest cut principle, then $C^{*}$ fully cuts $C_{k}$ whenever $C^{*}$ fully cuts $C_{k-1}$.

Proof Consider the cone-cutting $C_{k}=C_{k-1} \backslash\left(x_{(k)}\right)$. Since $\left(x_{(k)}\right)$ is selected by the lowest cut principle, then $\left(x_{(k)}\right)$ has the lowest cut-point on $C_{k-1}$. Therefore, there is no $C^{*}$-facet rejecting $V_{k-1}$ but accepting $V_{k}$. Otherwise, if there is a $C^{*}$-facet $\left(x_{j}\right)$ rejecting $V_{k-1}$ and accepting $V_{k}$, then $\left(x_{j}\right)$ breaks the line-segment $V_{k} V_{k-1}$. Let $Q$ be the intersection of $\left(x_{j}\right)$ on $V_{k} V_{k-1}$. Since $V_{k-1}$ is lower than $V_{k}$ and $Q$ is in between $V_{k}$ and $V_{k-1}, Q$ is lower than $V_{k}$. Since the cut-point of $\left(x_{j}\right)$ is the lowest real intersection of $\left(x_{j}\right)$, it is lower than $Q$. Hence the cut-point of $\left(x_{j}\right)$ is lower than the cut-point of $\left(x_{(k)}\right)$. This is a contradiction with the fact that $\left(x_{(k)}\right)$ has the lowest cut-point on $C_{k-1}$ among all cutters. Since there is no $C^{*}$-facet rejecting $V_{k-1}$ but accepting $V_{k}$, any red non- $C$-facet cuts off $V_{k}$ whenever it cuts off $V_{k-1}$. Therefore, $C^{*}$ fully cuts $C_{k}$ whenever $C^{*}$ fully cuts $C_{k-1}$.

Let $C_{0}$ be the basis cone of the original tableau $\left(T_{0}\right)$. A cone-renovation $C_{0} \rightarrow$ $C^{*}$ can be represented by simplex pivots started from an original tableau $\left(T_{0}\right)$ with index-set $J=J^{*} \cup\{d+1, \ldots, d+m\}$, where $J *=\operatorname{INDEX}\left(C^{*}\right)$ is known and $d=\left|J^{*} \backslash \operatorname{BASE}\left(T_{0}\right)\right|$, which is the number of red non- $C_{0}$ facets.

How to perform a cone-renovation $C \neq C^{*}$ by simplex pivots when $C \neq C_{0}$ ? Suppose while the simplex algorithm is being performed, at certain intermediate step the operator is told what the objective index set $J^{*}$ is. Suppose that the basis cone of the current tableau $(T)$ is the very cone $C$, then delete all indices $j \notin J^{*} \cup \operatorname{BASE}(T)$ from the available index-set $J$ and continue to take pivots toward the optimal solution. It represents the cone-renovation $C \rightarrow C^{*}$.

Theorem 3.1 If $C^{*}$ fully cuts a stable cone $C$, then the dual assembling conjecture is true. 
Proof The cone-renovation can be realized within $m$ steps of cone-cuttings/pivots.

In the beginning, $k=0, C_{0}=C$. Since $C^{*}$ fully cuts cone $C, C^{*}$ fully cuts cone $C_{0}$. According to Proposition 3.3, Since $C^{*}$ fully cuts stable cone $C_{0}, C^{*}$ uniformly cuts stable cone $C_{0}$. Select $\left(x_{(1)}\right)$ by (3.1), do cone-cutting and get cone $C_{1}=C_{0} \backslash\left(x_{(1)}\right)$. According to Point 2 of Proposition 2.3, since $C_{0}$. is stable, $C_{1}$ is stable. According to Proposition 3.2, since $C^{*}$ uniformly cuts stable cone $C_{0}$, there is no internal conflict on this cone-cutting, and the expelled facet $\left(x_{[i(1)]}\right)$ is black. Hence the index $[i(1)]$ can be deleted from the available index set $J$. According to Proposition 3.4, since the cutter $\left(x_{(1)}\right)$ is selected by (3.1) and since $C^{*}$ fully cuts $C_{0}, C^{*}$ fully cuts $C_{1}$.

For $k:=k+1$, select $\left(x_{(k)}\right)$ by (3.1), do cone-cutting and get cone $C_{k}=$ $C_{k-1} \backslash\left(x_{(k)}\right)$. According to Point 2 of Proposition 2.2, since $C_{k-1}$. is stable, $C_{k}$ is stable. According to Proposition 3.2, since $C^{*}$ uniformly cuts cone $C_{k-1}$, there is no internal conflict on this cone-cutting, and the expelled facet $\left(x_{[i(k)]}\right)$ is black. The index $[i(k)]$ can be deleted from the available index set $J$., According to Prop. 5.4, since the cutter $\left(x_{(k)}\right)$ is selected by (3.1) and since $C^{*}$ fully cuts $C_{k-1}, C^{*}$ fully cuts $C_{k}$.

Performing the same procedure repetitively, all red non-C facets will enter and stay in basis permanently. When the basis facets become all red facets, $C^{*}$ is obtained, The number of cone-cuttings taken in the procedure equals to the number of red non- $\mathrm{C}$ facets. The cone-renovation $C \rightarrow C^{*}$ can be realized within $m$ steps of cone-cuttings.

Theorem 3.1 could be seen as a conditional assembling conjecture. The condition is $C^{*}$ fully cuts $C$. If the condition is not satisfied, then the assembling conjecture may not be true. However, without explanation, the author has the following conjecture:

Relaxed assembling conjecture. The diameter of a $2 m$-facets polytope in $R^{m}$ is $m+a$, where ' $a$ ' is a constant being not larger than $m$.

\section{Gradient Falling, a Strongly Polynomial-Times' LP Algorithm?}

Motivated by the analysis on Hirsch conjecture, we are interested on the shortest path $\Lambda$ in the dual feasible region $D$, starting from a feasible point and ending at the lowest point $\boldsymbol{y}^{*}$. Imagine that $D$ is subjected as a smooth convex set, the shortest path $\Lambda \subseteq \mathrm{D}$ can be characterized as the follows: At each point $F \in \Lambda$, the direction vector $g$ along $\Lambda$ at $F$ has the maximal (normalized) inner product with the negative objective vector $-\boldsymbol{b}$ among all possible directions guiding an $\varepsilon>0$ path from $F$ within $D$, called the gradient direction at $F$ in $D$. When $D$ is not smooth, the shortest path is rather difficult to be described. We will introduce an algorithm named Gradient Falling in the section, which searches the lowest point $y^{*}$ along a broken line $\Lambda$; In each stage point $F$ of the broken line, the direction $g$ is the gradient direction at $F$, which has the maximal inner product with the negative objective vector $-\boldsymbol{b}$ among all possible directions within $D$. We will call such a broken line the falling path.

For ease, we use the symbol $\left(\boldsymbol{g}, \boldsymbol{\tau}_{j}\right)$ to represent the inner product of vectors $\boldsymbol{g}$ and $\boldsymbol{\tau}_{j}$. In this paper, $\boldsymbol{g}$ stands for a row vector and $\boldsymbol{\tau}_{j}$ stands for a column vector, the symbol $\left(\boldsymbol{g}, \boldsymbol{\tau}_{j}\right)$ employed here neglects the difference in between row vectors and column vectors. 
To realize the falling path, we only need to answer two questions: 1 . How to get the next stage point along a given gradient direction? 2 . How to get the gradient direction g?

\subsection{How to Get the Next Stage Point?}

Proposition 4.1 Given a ray $L=[F, F+\operatorname{tg})(t \geq 0)$, if $F \in D$ and there is no constraint plane $\left(x_{j}\right)$ passing through $F$ with $\left(\boldsymbol{g}, \boldsymbol{\tau}_{j}\right)<0$, then the next stage point is got as $F^{\prime}=F+t_{j *}$, where

$$
j^{*}:=\operatorname{argmin}_{j}\left\{t_{j}=\left(c_{j}-F \boldsymbol{\tau}_{j}\right) /\left(\boldsymbol{g}, \boldsymbol{\tau}_{j}\right) \mid\left(\boldsymbol{g}, \boldsymbol{\tau}_{j}\right)<0, t_{j}>0\right\}
$$

Proof Since the equation of $\left(x_{j}\right)$ is $\left(\boldsymbol{y}, \boldsymbol{\tau}_{j}\right)=c_{j}, F^{\prime}=F+t_{j} \boldsymbol{g}$, the intersection point of $\left(x_{j}\right)$ on ray $L$, satisfies that $\left(F+t_{j} \boldsymbol{g}, \boldsymbol{\tau}_{j}\right)=c_{j}$. The parameter can be determined by $t_{j}=\left(c_{j}-F \boldsymbol{\tau}_{j}\right) /\left(\boldsymbol{g}, \tau_{j}\right)$. If $\left(\boldsymbol{g}, \boldsymbol{\tau}_{j}\right)=0$, then there is no intersection; If $t_{j}<0$, then the intersection point is not in the ray $L$ but in its inversed ray. Therefore, the candidates $j$ for $j^{*}$ are required that $\left(\boldsymbol{g}, \boldsymbol{\tau}_{j}\right) \neq 0$ and $t_{j} \geq 0$. Since $F \in D, F$ is accepted by $\left(x_{j}\right): F \boldsymbol{\tau}_{j} \geq c_{j}$, i.e., $\left(c_{j}-F \boldsymbol{\tau}_{j}\right) \leq 0$, and then $\left(\boldsymbol{g}, \boldsymbol{\tau}_{j}\right)<0$. Since there is no constraint plane passing through $F$ with $\left(g, \tau_{j}\right)<0,\left(x_{j}\right)$ does not pass through $F$, and then $t_{j}>0$. Therefore, the candidates $j$ for $j^{*}$ are required that $\left(\boldsymbol{g}, \boldsymbol{\tau}_{j}\right)<0$ and $t_{j}>0$.

Sub-algorithm NextStagePoint $\left(F_{k-1}, \boldsymbol{g}\right)=F_{k}$

$F:=F_{k-1}$

$F:=F+t_{j^{*}} \boldsymbol{g}$ according to (4.1);

Output: $F_{k}=\operatorname{NextStagePoint}\left(F_{k-1}, \boldsymbol{g}\right)$..

Example 4.1 Given ${ }^{0} \boldsymbol{g}=-\boldsymbol{b}^{T}=(-4,-1,-4,-6,-2), F_{0}=(7,4,7,6,5) \in$ $D=\left\{\boldsymbol{y} \in Y^{5} \mid \boldsymbol{y}^{0} \boldsymbol{\tau}_{J} \geq c_{j}(j=1, \ldots, 9)\right\}$ :

${ }^{0} \boldsymbol{\tau}_{1}:=(2,0,0,1,1) ; \quad c_{1}:=1 ; \quad{ }^{0} \boldsymbol{\tau}_{2}:=(-1,1,2,1,0) ; \quad c_{2}:=1 ; \quad{ }^{0} \boldsymbol{\tau}_{3}:=$ $(0,1,0,1,1) ; \quad c_{3}:=2 ; \quad{ }^{0} \tau_{4}:=(-1,1,1,0,0) ; \quad c_{4}:=3 ;$

${ }^{0} \boldsymbol{\tau}_{5}:=(1,0,0,0,0) ;{ }^{0} \boldsymbol{\tau}_{6}:=(0,1,0,0,0) ;{ }^{0} \boldsymbol{\tau}_{7}:=(0,0,1,0,0) ;{ }^{0} \boldsymbol{\tau}_{8}:=$ $(0,0,0,1,0) ;{ }^{0} \tau_{9}:=(0,0,0,0,1) ; c_{5}:=c_{6}:=c_{7}:=c_{8}:=c_{9}:=0$;

$F:=F_{0} ; \boldsymbol{g}:={ }^{0} \boldsymbol{g}$;

$j=1 \quad t_{1}=\left(c_{1}-F \tau_{1}\right) /\left(g, \boldsymbol{\tau}_{1}\right)=(-27) /(-16)=27 / 16$

$j=2 \quad t_{2}=\left(c_{2}-F \boldsymbol{\tau}_{2}\right) /\left(\boldsymbol{g}, \boldsymbol{\tau}_{2}\right)=(-19) /(-11)=19 / 11$

$j=3 \quad t_{3}=\left(c_{3}-F \tau_{3}\right) /\left(g, \tau_{3}\right)=(-16) /(-9)=16 / 9$

$j=4 \quad t_{4}=\left(c_{4}-F \tau_{4}\right) /\left(g, \tau_{4}\right)=(-1) /(-1)=1$

$j=5 \quad t_{5}=\left(c_{5}-F \tau_{5}\right) /\left(g, \tau_{5}\right)=(-7) /(-4)=7 / 4$

$j=6 \quad t_{6}=\left(c_{6}-F \boldsymbol{\tau}_{6}\right) /\left(\boldsymbol{g}, \boldsymbol{\tau}_{6}\right)=(-4) /(-1)=4$

$j=7 \quad t_{7}=\left(c_{7}-F \tau_{7}\right) /\left(g, \tau_{7}\right)=(-7) /(-4)=7 / 4$

$j=8 \quad t_{8}=\left(c_{8}-F \tau_{8}\right) /\left(g, \boldsymbol{\tau}_{8}\right)=(-9) /(-6)=3 / 2$

$j=9 \quad t_{9}=\left(c_{9}-F \tau_{9}\right) /\left(g, \tau_{9}\right)=(-5) /(-2)=5 / 2$

$j *:=\operatorname{argmin}_{j}\left\{t_{j}=\left(c_{j}-F \boldsymbol{\tau}_{j}\right) /\left(\boldsymbol{g}, \boldsymbol{\tau}_{j}\right) \mid\left(\boldsymbol{g}, \boldsymbol{\tau}_{j}\right) \neq 0, t>0\right\}=\{4\} ; t_{4}=1$

$F_{1}:=F+g=(7,4,7,9,5)+(-4,-1,-4,-6,-2)=(3,3,3,3,3)$; 
NextStagePoint $\left(F_{0},{ }^{0} \boldsymbol{g}\right)=(3,3,3,3,3)$.

We are going to answer the second question: How to get the gradient direction at a stage point? We need to introduce a new concept, the projection of a vector in a cone.

\subsection{Projection of $\boldsymbol{g}$ in a Cone}

Let $L=(F, F+\operatorname{tg})(t>0)$ be a open ray started from $F$, and $\left(x_{j}\right)$ be a plane passing through $F$. We call $\boldsymbol{g}$ is lain in $\left(x_{j}\right)$ if $L \subseteq\left(x_{j}\right)_{\geq}=\left\{\boldsymbol{y} \mid\left(\boldsymbol{y}, \boldsymbol{\tau}_{j}\right) \geq c_{j}\right\}$ It is obvious that $\boldsymbol{g}$ is lain in $\left(x_{j}\right)$ if and only if $\left(\boldsymbol{g}, \boldsymbol{\tau}_{j}\right)=0$. Set $\Delta=\left\{j \mid\left(F, \boldsymbol{\tau}_{j}\right)=c_{j},\left(\boldsymbol{g}, \boldsymbol{\tau}_{j}\right) \leq 0\right\}, C=$ $\left\{\left(x_{j}\right)_{\geq} \mid j \in \Delta\right\}$.

Definition 4.1 The cone $C$ is called the door cone at $F,\left(x_{j}\right)$ is called a door at $F$ if $j \in \Delta$.

A door cone is a generalized cone. When the group of doors consists of $m$ independent planes, it is the regular cone mentioned in previous sections.

If $\left(x_{j}\right)$ is a door with $\left(\boldsymbol{g}, \boldsymbol{\tau}_{j}\right)<0$ at $F$, then whole the open ray $L$ is rejected by $\left(x_{j}\right)$. In this case, the falling path is blocked by the door $\left(x_{j}\right)$ and the gradient direction $\boldsymbol{g}$ must be changed to its projection in $\left(x_{j}\right)$. It is known that the projection of $\boldsymbol{d}$ in plane $\left(x_{j}\right)$ can be calculated as follows:

$$
\boldsymbol{d}_{\downarrow j}=\boldsymbol{d}-\left[\left(\boldsymbol{d}, \boldsymbol{\tau}_{j}\right) /\left(\boldsymbol{\tau}_{j}, \boldsymbol{\tau}_{j}\right)\right] \boldsymbol{\tau}_{j}
$$

Projection of $\boldsymbol{d}$ in $\left(x_{j}\right)$ has the maximal inner product with the vector $\boldsymbol{d}$ among all directions lain in $\left(x_{j}\right)$. It is obvious that $\boldsymbol{d}_{\downarrow j}=\mathbf{0}$ if and only if there is a constant $c$ such that $\boldsymbol{d}=c \boldsymbol{\tau}_{j}$. i.e., $\boldsymbol{d}$ is vertical to $\left(x_{j}\right)$. We call $\boldsymbol{d}_{\downarrow j}$ a zero projection if $\boldsymbol{d}_{\downarrow j}=\mathbf{0}$. When the gradient direction is zero, there is no way to go along the full path down-ward. The target of optimal searching is to find out where the gradient vector becomes to zero.

Since that $\left(\boldsymbol{d}_{\downarrow j}, \boldsymbol{\tau}_{j}\right)=\left(\boldsymbol{d}, \boldsymbol{\tau}_{j}\right)-\left[\left(\boldsymbol{d}, \boldsymbol{\tau}_{j}\right) /\left(\boldsymbol{\tau}_{j}, \boldsymbol{\tau}_{j}\right)\right]\left(\boldsymbol{\tau}_{j}, \boldsymbol{\tau}_{j}\right)=\left(\boldsymbol{d}, \boldsymbol{\tau}_{j}\right)-\left(\boldsymbol{d}, \boldsymbol{\tau}_{j}\right)=$ 0 , the projection $\boldsymbol{d}_{\downarrow j}$ lies in $\left(x_{j}\right)$ always. When there is only one door $\left(x_{j}\right)$ blocks the way, $\boldsymbol{g}_{\downarrow(j)}$ is the gradient direction at $F$. But when there are more than one block doors at a stage point, how to get a projected direction lain in those blockers both?

Let $\boldsymbol{d}_{\downarrow 12}=\boldsymbol{d}_{\downarrow 1}-\left[\left(\boldsymbol{d}_{\downarrow 1}, \boldsymbol{\tau}_{2}\right) /\left(\boldsymbol{\tau}_{2}, \boldsymbol{\tau}_{2}\right)\right] \boldsymbol{\tau}_{2}$ be the projection of $\boldsymbol{d}_{\downarrow 1}$ in $\left(x_{2}\right)$. Which lies in $\left(x_{2}\right)$ of course, but it lies in $\left(x_{1}\right)$ if and only if $\left(\boldsymbol{\tau}_{1}, \boldsymbol{\tau}_{2}\right)=0$. We can not get the projection of $\boldsymbol{g}$ in $\left(x_{2}\right) \cap\left(x_{2}\right)$ by successively doing projections in $\left(x_{2}\right)$ and $\left(x_{2}\right)$ whenever $\left(\boldsymbol{\tau}_{1}, \boldsymbol{\tau}_{2}\right) \neq 0$.

The author does not know any existent knowledge about projection of a vector in the intersections of two or more planes, but tray to give definitions and propositions himself as follows.

Definition 4.2 Given a direction $\boldsymbol{g}$ and a door cone $C$, vector $\boldsymbol{g}_{\downarrow}$ is called the projection of $\boldsymbol{g}$ in door cone $C$, if, among the vectors $F+\boldsymbol{d} \in C$, it has maximal normalized inner product with $g$ :

$$
\boldsymbol{g}_{\underline{c}}=\operatorname{argmax}_{d}\left\{(\boldsymbol{d}, \boldsymbol{g}) /(\boldsymbol{d}, \boldsymbol{d}) \mid\left(\boldsymbol{d}, \boldsymbol{\tau}_{j}\right)=0 \text { for } j \in \Delta\right\} .
$$


To get projection of $g$ in $C$, we need to do (dual) eliminations. Let $(E)$ be the coefficient matrix of $\left(x_{(1)}\right), \ldots,\left(x_{(r)}\right)$ (No need to including constant row in the simplex tableau). The procedure of elimination is a mapping

$$
{ }^{i} p:{ }^{i-1} Y \rightarrow{ }^{i} Y:{ }^{i} p\left({ }^{i-1} \boldsymbol{y}=\left(y_{\langle i\rangle}, \ldots, y_{\langle m\rangle}\right)\right)={ }^{i} \boldsymbol{y}=\left(y_{\langle i+1\rangle}, \ldots, y_{\langle m\rangle}\right) .
$$

Mapping ${ }^{i} p$ keeps the coordinates of a point invariable for all components except hiding the component $y_{\langle i\rangle}$. Neglecting the index $i$, mapping $p$ deletes the first component with respect to the order $\langle i\rangle$. After $m$ times of elimination, we get theeliminations tableau $(E *)$ :

\begin{tabular}{llllllll}
\hline & $\left(x_{(1)}\right)$ & $\left(x_{(2)}\right)$ & $\left(x_{(2)}\right)$ & $\ldots$ & $\left(x_{(k)}\right)$ & $\ldots$ & $\left(x_{(m)}\right)$ \\
\hline$y_{\langle 1\rangle}$ & ${ }^{0} \tau_{\langle 1\rangle(1)}$ & $0 \ldots$ & 0 & $\ldots$ & 0 & $\ldots$ & 0 \\
$y_{\langle 2\rangle}$ & ${ }^{0} \tau_{\langle 2\rangle(1)}$ & ${ }^{1} \tau_{\langle 2\rangle(2)}$ & 0 & & 0 & 0 & 0 \\
$y_{\langle 3\rangle}$ & ${ }^{0} \tau_{\langle 3\rangle(1)}$ & ${ }^{1} \tau_{\langle 3\rangle(2)}$ & ${ }^{1} \tau_{\langle 3\rangle(2)}$ & & 0 & 0 & 0 \\
& & $\ldots$ & $\ldots$ & & & & \\
$y_{\langle m\rangle}$ & ${ }^{0} \tau_{\langle m\rangle(1)}$ & ${ }^{1} \tau_{\langle m\rangle(2)}$ & & & $\tau_{\langle k\rangle(k)}$ & $\ldots$ & ${ }^{k-1} \tau_{\langle m\rangle(k)}$ \\
\hline
\end{tabular}

The tableau is got by doing preliminary column-transformations successively to make all elements in the upper-right triangle to be zero. The index stood on the left shoulder of elements shows the number of hidden components. In the first row, the number is zero, it means that the first plane has its original coefficients: ${ }^{0} \boldsymbol{\tau}_{(1)}=\boldsymbol{\tau}_{(1)}$ and it is a $m$-dimensional vector; The hidden variables' number on the left shoulder of elements in the $j$ th column is $j-1$, it means that the vector ${ }^{j-1} \boldsymbol{\tau}_{(j)}=\left({ }^{j-1} \tau_{\langle j\rangle(j)}, \ldots,{ }^{j-1} \tau_{\langle m\rangle(j)}\right)^{T}$ is not original, but got after $j-1$ time of eliminations-transformations, it is the coefficient vector of $\left(x_{(1)}\right) \cap \ldots \cap\left(x_{(j)}\right)$ (not only $\left(x_{(j)}\right)$ !), a $(m-j+1)$-dimensional vector. Suppose that $r<m,{ }^{r-1} \boldsymbol{\tau}_{(r)}$ is called the solution vector with respect to door cone $C_{r}$. The solution of the $r$ linear equations can be gathered at the solution space ${ }^{r-1} Y=\left\{{ }^{r-1} \boldsymbol{y}=\left(y_{\langle r\rangle}, \ldots, y_{\langle m\rangle}\right)\right\}$ : For arbitrary vector $^{r-1} \boldsymbol{y} \in^{r-1} Y$, there determines a solution of the equations $\boldsymbol{y}^{*}=\left(y_{1}, \ldots, y_{m}\right)$, called the hidden solution of ${ }^{r-1} \boldsymbol{y}$ got by the algorithm as follows:

Sub-algorithm HiddenSolution(y) (Requirement: $\boldsymbol{y} \in^{r-1} Y$ )

Input: $\boldsymbol{y}=\left(y_{\langle r\rangle}, \ldots, y_{\langle m\rangle}\right)$

If $r=1$, then Hidden Solution $(\boldsymbol{y})=\boldsymbol{y}$; Else

For $s=1$ to $r-1$,

$$
\begin{aligned}
& u:=-\left(1 /{ }^{r-s-1} \tau_{\langle r-s\rangle(r-s)}\right)\left({ }^{r-s} p\left({ }^{r-s-1} \boldsymbol{\tau}_{r-s}\right),{ }^{r-s} \boldsymbol{y}\right) ; \quad\left({ }^{r-s} p(\boldsymbol{\tau})\right. \\
& \quad \text { deletes the first component from } \boldsymbol{\tau}) \\
& { }^{r-s-1} \boldsymbol{y}:=\left(u,{ }^{r-s} \boldsymbol{y}\right)=\left(u,{ }^{r-s} d_{\langle r-s+1\rangle}, \ldots,{ }^{r-s} d_{\langle m\rangle}\right) ;
\end{aligned}
$$

Output: HiddenSolution $(\boldsymbol{y})={ }^{0} \boldsymbol{y}$. 
The representation of the procedure (4.4) is trivial, but it is very easy and acquainted by people in resolving linear equations.

Proposition 4.2 Let $\boldsymbol{d}_{\downarrow}$ be the projection of $\boldsymbol{d}$ in $\left(x_{1}\right)$, among the directions lain in $\left(x_{1}\right)$, the one has maximal inner product with $\boldsymbol{d}$ if and only if it has maximal inner product with $\boldsymbol{d}_{\downarrow}$.

Proof If $\boldsymbol{y}$ is a direction lain in $\left(x_{1}\right)$, then $\left(\boldsymbol{\tau}_{1}, \boldsymbol{y}\right)=0$. Since $\boldsymbol{d}_{\downarrow}=\boldsymbol{d}-\left[\left(\boldsymbol{d}, \boldsymbol{\tau}_{1}\right) /\right.$ $\left.\left(\boldsymbol{\tau}_{1}, \boldsymbol{\tau}_{1}\right)\right] \tau_{1},\left(\boldsymbol{d}_{\downarrow}, \boldsymbol{y}\right)=(\boldsymbol{d}, \boldsymbol{y})-\left[\left(\boldsymbol{d}, \boldsymbol{\tau}_{1}\right) /\left(\boldsymbol{\tau}_{1}, \boldsymbol{\tau}_{1}\right)\right]\left(\boldsymbol{\tau}_{1}, \boldsymbol{y}\right)=(\boldsymbol{d}, \boldsymbol{y})$. The proposition is true.

For any $i<m$, denote ${ }^{i} \boldsymbol{d}=\left(d_{\langle i+1\rangle}, \ldots, d_{\langle m\rangle}\right)$.

Proposition 4.3 The projection of ${ }^{i} \boldsymbol{d}=\left(d_{\langle i+1\rangle}, \ldots, d_{\langle m\rangle}\right)$ in ${ }^{m-i} Y$ is ${ }^{i} \boldsymbol{d}_{\downarrow}=$ $\left(0, d_{\langle i+2\rangle}, \ldots, d_{\langle m\rangle}\right)$, and ${ }^{i} p\left({ }^{i} \boldsymbol{d}_{\downarrow}\right)={ }^{i} \boldsymbol{p}\left({ }^{i} \boldsymbol{d}\right)={ }^{i+1} \boldsymbol{d}$.

Proof The coefficient vector of ${ }^{m-i} Y$ is ${ }^{i} \tau=\left({ }^{i} \tau_{\langle i+1\rangle}=1,0, \ldots, 0\right)$. We have that

$$
\begin{aligned}
& { }^{i} \boldsymbol{d}_{\downarrow}={ }^{i} \boldsymbol{d}-\left[\left({ }^{i} \boldsymbol{d}, \boldsymbol{\tau}\right) /\left({ }^{i} \boldsymbol{\tau},{ }^{i} \boldsymbol{\tau}\right)\right]{ }^{i} \boldsymbol{\tau}=\left(d_{\langle i+1\rangle}, \ldots, d_{\langle m\rangle}\right)-\left(d_{\langle i+1\rangle}\right) /(1)(1,0, \ldots, 0) \\
& \quad=\left(0, d_{\langle i+2\rangle}, \ldots, d_{\langle m\rangle}\right) ; \\
& { }^{i} p\left({ }^{i} \boldsymbol{d}_{\downarrow}\right)={ }^{i} \boldsymbol{p}\left({ }^{i} \boldsymbol{d}\right)=\left(d_{\langle i+2\rangle}, \ldots, d_{\langle m\rangle}\right)={ }^{i+1} \boldsymbol{d} .
\end{aligned}
$$

Proposition 4.4 Let ${ }^{\cdots i} p(\boldsymbol{d})={ }^{1} p_{\circ} \ldots{ }^{i} p(\boldsymbol{d})={ }^{i} p\left[\ldots\left[{ }^{1} p(\boldsymbol{d})\right]\right]$ be the composition of elimination mappings, we have that

$$
{ }^{\cdots i} p(\boldsymbol{d})={ }^{i} \boldsymbol{d} \quad(i<m) .
$$

Proof This is the direct corollary of Proposition 4.3.

Theorem 4.1 The projection of vector $\boldsymbol{d}$ in the door cone $C$ is the hidden solution of ${ }^{r-1} \boldsymbol{d}_{\downarrow}={ }^{r-1} \boldsymbol{d}-\left[\left({ }^{r-1} \boldsymbol{d},{ }^{r-1} \boldsymbol{\tau}_{(r)}\right) /\left({ }^{r-1} \boldsymbol{\tau}_{(r)},{ }^{r-1} \boldsymbol{\tau}_{(r)}\right)\right]^{r-1} \boldsymbol{\tau}_{(r)}$, i.e.

$$
\boldsymbol{d}_{\downarrow c}=\text { HiddenSolution }\left({ }^{r-1} \boldsymbol{d}_{\downarrow}\right)
$$

where $^{r-1} \boldsymbol{\tau}_{(r)}$ is the solution vector with respect to $C$.

Proof Since ${ }^{r-1} \boldsymbol{\tau}_{(r)}$ is the equation of $\left(x_{(1)}\right) \cap \cdots \cap\left(x_{(r)}\right)$ in ${ }^{r-1} Y$, the vector ${ }^{r-1} \boldsymbol{d}_{\downarrow}=$ ${ }^{r-1} \boldsymbol{d}-\left[\left({ }^{r-1} \boldsymbol{d},{ }^{r-1} \boldsymbol{\tau}_{(r)}\right) /\left({ }^{r-1} \boldsymbol{\tau}_{(r)},{ }^{r-1} \boldsymbol{\tau}_{(r)}\right)\right]^{r-1} \boldsymbol{\tau}_{(r)}$ is the projection of ${ }^{r-1} \boldsymbol{d}$ in $\left(x_{(1)}\right) \cap$ $\cdots \cap\left(x_{(r)}\right)$ within the subspace space ${ }^{r-1} Y$. It lies in ${ }^{r-1} Y$ certainly. By means of Proposition $4.4,{ }^{r-1} \boldsymbol{d}=\cdots(r-1) p(\boldsymbol{d})$, which is the composition of projections from $\boldsymbol{d}={ }^{0} \boldsymbol{d}$. According to Proposition 4.2, the composition of projection equals to the directed projection from $\boldsymbol{d}$ to $\left(x_{(1)}\right) \cap \cdots \cap\left(x_{(r)}\right)$. Therefore ${ }^{r-1} \boldsymbol{d}_{\downarrow}$ is the projection of $\boldsymbol{d}$ in cone $C$, but it is expressed in the sub-space ${ }^{r-1} Y$. Since that ${ }^{r-1} \boldsymbol{d}_{\downarrow} \in \in^{r-1} Y$, the requirement of formula (4.6) is satisfied, solution $\boldsymbol{d}_{\underline{c}}=\operatorname{Hidden\operatorname {Solution}}\left({ }^{r-1} \boldsymbol{d}_{\downarrow}\right)$ is the projection of $\boldsymbol{d}$ in $C$ represented in the original space $Y$. 
The vector ${ }^{r-1} \boldsymbol{d}_{\downarrow}$ occurring in theorem is called the intersected projection of $\boldsymbol{d}$. The theorem tells us how to get the projection of $\boldsymbol{d}$ in $\left(x_{(1)}\right) \cap \cdots \cap\left(x_{(r)}\right)$. See the Example 4.1:

\begin{tabular}{lllllll}
\hline$(E)$ & $\tau_{1}$ & $\tau_{2}$ & $\tau_{3}$ & $\tau_{4}$ & $\tau_{5}$ & $d$ \\
\hline$y_{1}$ & 2 & -1 & 0 & -1 & 1 & -4 \\
$y_{4}$ & 0 & 1 & 1 & 1 & 0 & -1 \\
$y_{2}$ & 0 & 2 & 0 & 1 & 0 & -4 \\
$y_{3}$ & 1 & 1 & 1 & 0 & 0 & -6 \\
$y_{5}$ & 1 & 0 & 1 & 0 & 0 & -2 \\
\hline$\left(E^{*}\right)$ & 0 & 1 & & & & \\
& ${ } \tau_{1}$ & $\tau_{2}$ & ${ }^{2}$ & $\tau_{3}$ & $4 \tau_{5}$ & $d$ \\
\hline$y_{1}$ & $\mathbf{2}$ & 0 & 0 & 0 & 0 & -4 \\
$y_{4}$ & 0 & $\mathbf{1}$ & 0 & 0 & 0 & -1 \\
$y_{2}$ & 0 & 2 & $-\mathbf{2}$ & 0 & 0 & -4 \\
$y_{3}$ & 1 & $3 / 2$ & $-1 / 2$ & $-\mathbf{3 / 4}$ & 0 & -6 \\
$y_{5}$ & 1 & $1 / 2$ & $1 / 2$ & $-1 / 4$ & $-\mathbf{1 / 3}$ & -2 \\
\hline
\end{tabular}

$r=1$ : The solution vector is ${ }^{r-1} \boldsymbol{\tau}_{(k)}={ }^{0} \boldsymbol{\tau}_{(1)}=(2,0,0,1,1),{ }^{0} \boldsymbol{d}=(-4,-1,-4$, $-6,-2)$,

$$
\begin{aligned}
{ }^{0} \boldsymbol{d}_{\downarrow} & ={ }^{0} \boldsymbol{d}-\left[\left({ }^{0} \boldsymbol{d},{ }^{0} \boldsymbol{\tau}_{(1)}\right) /\left({ }^{0} \boldsymbol{\tau}_{(1)},{ }^{0} \boldsymbol{\tau}_{(1)}\right)\right]{ }^{0} \boldsymbol{\tau}_{(1)} \\
& =(-4,-1,-4,-6,-2)-[(-16) /(6)](2,0,0,1,1) \\
& =(4 / 3,-1,-4,-10 / 3,2 / 3)
\end{aligned}
$$

$\boldsymbol{d}_{\downarrow c}=\boldsymbol{d}_{\downarrow 1}=$ HiddenSolution $\left({ }^{0} \boldsymbol{d}_{\downarrow}\right)=(4 / 3,-1,-4,-10 / 3,2 / 3)$, which is the very projection of $\boldsymbol{d}$ in $\left(x_{1}\right)$.

$r=2$ : The solution vector is ${ }^{1} \boldsymbol{\tau}_{(2)}=(1,2,3 / 2,1 / 2),{ }^{1} \boldsymbol{d}=(-1,-4,-6,-2)$,

$$
\begin{aligned}
{ }^{1} \boldsymbol{d}_{\downarrow} & ={ }^{1} \boldsymbol{d}-\left[\left({ }^{1} \boldsymbol{d},{ }^{1} \boldsymbol{\tau}_{2}\right) /\left({ }^{1} \boldsymbol{\tau}_{2},{ }^{1} \boldsymbol{\tau}_{2}\right)\right]{ }^{1} \boldsymbol{\tau}_{2} \\
& =(-1,-4,-6,-2)-[(-19) /(30 / 4)](2 / 2,4 / 2,3 / 2,1 / 2) \\
& =(23 / 15,16 / 15,-33 / 15,-11 / 15)
\end{aligned}
$$

$\boldsymbol{d}_{\downarrow c}=\boldsymbol{d}_{\downarrow 12}=$ HiddenSolution $\left({ }^{1} \boldsymbol{d}_{\downarrow}\right)=(22 / 15,23 / 15,16 / 15,-33 / 15,-11 / 15)$.

Where the hidden value of $\boldsymbol{d}_{\downarrow 12}$ is got by (4.4) for $s=r-1=1$ :

$$
\begin{aligned}
u: & =-\left(1 /{ }^{r-s-1} \tau_{\langle r-s\rangle(r-s)}\right)\left({ }^{r-s} p\left({ }^{r-s-1} \tau_{r-s}\right),{ }^{r-s} \boldsymbol{d}\right) \\
& =-\left(1 /{ }^{1} \tau_{\langle 2\rangle(2)}\right)\left({ }^{1} p\left({ }^{0} \boldsymbol{\tau}_{1}\right),{ }^{1} \boldsymbol{d}\right)= \\
& =-(1 / 2)((0,0,1,1),(23 / 15,16 / 15,-33 / 15,-11 / 15)) \\
& =22 / 15 .
\end{aligned}
$$


Checking: $\left(\boldsymbol{d}_{\downarrow 12},{ }^{0} \boldsymbol{\tau}_{1}\right)=((22 / 15,23 / 15,16 / 15,-33 / 15,-11 / 15),(2,0,0,1,1))=$ 0 ;

$$
\left(\boldsymbol{d}_{\downarrow 12},{ }^{0} \boldsymbol{\tau}_{2}\right)=((22 / 15,23 / 15,16 / 15,-33 / 15,-11 / 15),(-1,1,2,1,0))=0 ;
$$

It shows $\boldsymbol{d}_{\downarrow 12}$ lies in $\left(x_{1}\right) \cap\left(x_{2}\right)$, which is the projection of $\boldsymbol{d}$ in $\left(x_{1}\right) \cap\left(x_{2}\right)$.

The projections of $\boldsymbol{g}$ in cone are commutative. To show this property, let's set a new order of $\Delta=\{1,2,3,4,5\}$ for example. Set

$$
(1):=2 ;(2):=1 ;(j):=j:(j \neq 1,2)
$$

i.e., take a permutation in between the first and the second columns in tableau $(E)$. To get its elimination matrix, the diagonal elements must be non-zero, we need to exchange the 2-th and 4-th rows on the tableau:

$$
\langle 2\rangle:=4 ;\langle 3\rangle:=2 ;\langle 4\rangle:=3 ;\langle 4\rangle:=2 ;\langle 3\rangle:=k:(k \neq 2,4) .
$$

We get the tableau $\left(E^{\prime}\right)$ and its elimination tableau $\left(E^{*^{\prime}}\right)$ as follows:

\begin{tabular}{lclllll}
\hline$\left(E^{\prime}\right)$ & $\tau_{2}$ & $\tau_{1}$ & $\tau_{3}$ & $\tau_{4}$ & $\tau_{5}$ & \\
\hline$y_{1}$ & -1 & 2 & 0 & -1 & 1 & -4 \\
$y_{4}$ & 1 & 1 & 0 & 0 & 1 & -6 \\
$y_{2}$ & 1 & 1 & 1 & 0 & 1 & -1 \\
$y_{3}$ & 2 & 0 & 1 & 1 & 0 & -4 \\
$y_{5}$ & 0 & 0 & 0 & 0 & 0 & -2 \\
\hline$\left(E^{*^{\prime}}\right)$ & 0 & & & & & \\
& $\tau_{2}$ & 1 & $2 \tau_{3}$ & ${ }^{2} \tau_{4}$ & $4 \tau_{5}$ & \\
\hline$y_{1}$ & $-\mathbf{1}$ & 0 & 0 & 0 & 0 & -4 \\
$y_{4}$ & 1 & $\mathbf{3}$ & 0 & 0 & 0 & -6 \\
$y_{2}$ & 1 & 2 & $\mathbf{1 / 3}$ & 0 & 0 & -1 \\
$y_{3}$ & 2 & 4 & $-4 / 3$ & $\mathbf{3}$ & 0 & -4 \\
$y_{5}$ & 0 & 1 & 1 & -2 & 0 & -2 \\
\hline
\end{tabular}

$r=2:$ The solution vector is ${ }^{1} \boldsymbol{\tau}_{(1)}=(3,2,4,1),{ }^{1} \boldsymbol{d}=(-6,-1,-4,-2)$,

$$
\begin{aligned}
{ }^{1} \boldsymbol{d}_{\downarrow} & \left.={ }^{1} \boldsymbol{d}-\left[{ }^{1} \boldsymbol{d},{ }^{1} \boldsymbol{\tau}_{(2)}\right) /\left({ }^{1} \boldsymbol{\tau}_{(2)},{ }^{1} \boldsymbol{\tau}_{(2)}\right)\right]{ }^{1} \boldsymbol{\tau}_{(2)} \\
& =(-6,-1,-4,-2)-[(-38) /(30)](3,2,4,1) \\
& =(-33 / 15,23 / 15,16 / 15,-11 / 15)
\end{aligned}
$$

$\boldsymbol{d}_{\downarrow(1)(2)}=$ HiddenSolution $\left({ }^{1} \boldsymbol{d}_{\downarrow}\right)=(22 / 15,-33 / 15,23 / 15,16 / 15,-11 / 15)$. 
Don't forget to change it back to the original orders: According to (4.7) and (4.8), we get that

$$
\begin{aligned}
\boldsymbol{d}_{\downarrow 21} & =\boldsymbol{d}_{\downarrow(1)(2)}=\left(d_{\langle k\rangle} \mid\langle k\rangle=1,2,3,4,5\right) \\
& =(22 / 15,23 / 15,16 / 15,-33 / 15,-11 / 15) .
\end{aligned}
$$

Comparing (*) with (**), we have checked that $\boldsymbol{d}_{\downarrow 21}=\boldsymbol{d}_{\downarrow 12}$, the projections in $C_{r}$ are commutative.

We are going to introduce our algorithm on falling path now. From a stage point $F$, determining the index set $\Delta$ of doors at $F$, we can get the elimination matrix $E^{*}$, and get the projection $\boldsymbol{g}$ on door cone $C$. If $\boldsymbol{g}=\mathbf{0}$, then $F$ is the lowest point $V^{*}$, Else, we can use the sub-algorithm $F:=\operatorname{NextStagePoint}(F, g)$ to renew the stage point. Repeat the process until the gradient vector $g$ degenerates to zero. This is the core idea of our algorithm.

\subsection{Algorithm GradientFalling $\left({ }^{0} T, F_{0},{ }^{0} \mathbf{g}\right)$}

Input: Simplex tableau $\left({ }^{0} T\right) F_{0},{ }^{0} \boldsymbol{g}:=-\boldsymbol{b}^{T}$;

$(1):=1 ; \ldots ;(m+n):+m+n ;\langle 1\rangle:=1 ; \ldots ;\langle m\rangle:=m$;

$k:=0$

$\Delta:=\left\{j \mid\left(F_{0},{ }^{0} \boldsymbol{\tau}_{j}\right)={ }^{0} c_{j},\left({ }^{0} \boldsymbol{g},{ }^{0} \boldsymbol{\tau}_{j}\right) \leq 0\right\} ;$

If $\Delta=\varnothing$, then go to step 3;

Else, $k:=k+1$; go to step 1 ;

For $k=1$ to $m+n$

Step 1. Determining the elimination matrix for cone $C_{k}$ :

If ${ }^{k-1} \tau_{\langle k\rangle(k)}=0$, then $\langle k\rangle:=$ the first non-zero index $i ;\langle i\rangle:=k$;

Doing dual elimination operation at eye $(k, k)$ on $\left({ }^{k-1} T\right)$ to get $\left({ }^{k} T\right)$;

Step 2. Getting gradient direction $\underline{\boldsymbol{g}}_{\downarrow \ldots(k)}$ at $F_{k}$ :

Calculate intersected projection ${ }^{k} \boldsymbol{d}_{\downarrow}:={ }^{k-1} \boldsymbol{g}-\left[\left({ }^{k-1} \boldsymbol{g},{ }^{k} \boldsymbol{\tau}_{(k+1)}\right) /\right.$

$\left.\left({ }^{k} \boldsymbol{\tau}_{(k+1)},{ }^{k} \boldsymbol{\tau}_{(k+1)}\right)\right]^{k} \boldsymbol{\tau}_{(k+1)} ;$

$\underline{\boldsymbol{g}}_{\downarrow \ldots(k)}=$ HiddenSolution $\left({ }^{k} \boldsymbol{d}_{\downarrow}\right)=\left({ }^{k} g_{\langle 1\rangle}, \ldots,{ }^{k} g_{\langle m\rangle}\right)$;

Return the components in original order; $\underline{g}_{\downarrow \ldots(k)}:=\left({ }^{k} g_{1}, \ldots,{ }^{k} g_{m}\right)$;

If $\underline{\boldsymbol{g}}_{\downarrow \ldots(k)}=\mathbf{0}$, then stop and output, $V^{*}=F_{k}$;

Step 3. Getting next stage point and the door cone $C_{k+1}$ :

$F_{k+1}=\operatorname{NextStagePoint}\left(F_{k}, \underline{\boldsymbol{g}}_{\downarrow \ldots(k)}\right) ; \quad\left(\underline{\boldsymbol{g}}_{\downarrow \ldots(k)}={ }^{0} \boldsymbol{g}\right.$ when $\left.k=0\right)$

$\left.\Delta:=\left\{j \mid\left(F_{k},{ }^{0} \boldsymbol{\tau}_{j}\right)=c_{j}, \underline{\boldsymbol{g}}_{\downarrow \ldots(k)}, \boldsymbol{\tau}_{j}\right) \leq 0\right\} ;$

Renew the order of indices keeping priority to $\Delta$-indices

(i.e., $(j)<\left(j^{\prime}\right)$, if $(j) \in \Delta$ and $\left.\left(j^{\prime}\right) \notin \Delta\right)$

$k:=k+1$; Go back to step 1 ;

Return to Example 4.1. 


\begin{tabular}{llrlrllllll}
\hline$\left({ }^{0} T\right)$ & $\tau_{1}$ & $\tau_{2}$ & $\tau_{3}$ & $\tau_{4}$ & $\tau_{5}$ & $\tau_{6}$ & $\tau_{7}$ & $\tau_{8}$ & $\tau_{9}$ & $\mathrm{~g}$ \\
\hline$y_{1}$ & 2 & -1 & 0 & -1 & 1 & 0 & 0 & 0 & 0 & -4 \\
$y_{2}$ & 0 & 1 & 1 & 1 & 0 & 1 & 0 & 0 & 0 & -1 \\
$y_{3}$ & 0 & 2 & 0 & 1 & 0 & 0 & 1 & 0 & 0 & -4 \\
$y_{4}$ & 1 & 1 & 1 & 0 & 0 & 0 & 0 & 1 & 0 & -6 \\
$y_{5}$ & 1 & 0 & 1 & 0 & 0 & 0 & 0 & 0 & 1 & -2 \\
\hline
\end{tabular}

$(1):=1, \ldots(9):=9 ;\langle 1\rangle:=1 ; \ldots ;\langle 5\rangle:=5 ;$

$k=0 . \Delta=\varnothing$, there is no door at $F_{0}=(7,4,7,6,5)$, go to step 3,

Step 3 Getting next stage point and the door cone $C_{1}$ :

$$
\begin{aligned}
F_{1} & =F_{k+1}=\operatorname{NextStagePoint}\left(F_{k}, \underline{\boldsymbol{g}}_{\downarrow \ldots(k)}\right)=\operatorname{NextStagePoint}\left(F_{0},{ }^{0} \boldsymbol{g}\right)=(3,3,3,3) ; \\
\Delta & :=\left\{j \mid\left(F_{0},{ }^{0} \boldsymbol{\tau}_{j}\right)=c_{j},\left({ }^{0} \boldsymbol{g},{ }^{0} \boldsymbol{\tau}_{j}\right) \leq 0\right\}=\{4\} ; \\
(1) & :=4 ;(4):=1 ; \text { (from now on, the order in index-set } J \text { is } \\
J & =(4,1,2,3,5,6,7,8,9))
\end{aligned}
$$

$k=1$.

Step 1. Determining elimination matrix for cone $C_{1}$ :

Since ${ }^{0} \tau_{\langle 1\rangle(1)} \neq 0$, doing elimination operation at eye $(1,1)$ on $\left({ }^{0} T\right)$, get $\left({ }^{1} T\right)$ :

\begin{tabular}{lrlllllllll}
\hline & $\tau_{4}$ & $\tau_{2}$ & $\tau_{3}$ & $\tau_{1}$ & $\tau_{5}$ & $\tau_{6}$ & $\tau_{7}$ & $\tau_{8}$ & $\tau_{9}$ & $\mathrm{~g}$ \\
\hline$y_{1}$ & $\mathbf{- 1}$ & 0 & 0 & 0 & 0 & 0 & 0 & 0 & 0 & -4 \\
$y_{2}$ & 1 & 0 & 1 & 2 & 1 & 1 & 0 & 0 & 0 & -1 \\
$y_{3}$ & 1 & 1 & 0 & 2 & 1 & 0 & 1 & 0 & 0 & -4 \\
$y_{4}$ & 0 & 1 & 1 & 1 & 0 & 0 & 0 & 1 & 0 & -6 \\
$y_{5}$ & 0 & 0 & 1 & 1 & 0 & 0 & 0 & 0 & 1 & -2 \\
\hline
\end{tabular}

The sub-matrix including left 1-th column forms the elimination-matrix $E^{*}$ with respect to $C_{1}$.

Step 2: Getting the gradient direction $\underline{\boldsymbol{g}}_{\downarrow(1)}$ at $F_{1}$ :

The solution vector of $E^{*}$ is ${ }^{k-1} \boldsymbol{\tau}_{(k)}={ }^{0} \boldsymbol{\tau}_{(1)}=(-1,1,1,0,0)$, Calculate the intersection projection

$$
\begin{gathered}
\begin{array}{c}
{ }^{1} \boldsymbol{d}_{\downarrow}:=(-4,-1,-4,-6,-2)-(-1) /(3)(-1,1,1,0,0) \\
=(-13 / 3,-2 / 3,-11 / 3,-6,-2) ;
\end{array} \\
\underline{\boldsymbol{g}}_{\downarrow(1)}:=\text { HiddenSolution }\left({ }^{1} \boldsymbol{d}_{\downarrow}\right)=(-13 / 3,-2 / 3,-11 / 3,-6,-2) ;
\end{gathered}
$$


Return to original order, get $\underline{g}_{\downarrow 4}=(-13 / 3,-2 / 3,-11 / 3,-6,-2)$;

Step 3 Getting next stage point and the door cone $C_{2}$ :

$$
\begin{aligned}
F_{2} & =\operatorname{NextStagePoint}\left(F_{1}, \underline{\boldsymbol{g}}_{\downarrow 4}\right)=(5 / 6,8 / 3,7 / 6,0,2) \\
\Delta: & =\left\{j \mid\left(F_{2},{ }^{0} \boldsymbol{\tau}_{j}\right)=c_{j},\left(\underline{\boldsymbol{g}}_{\downarrow(1)},{ }^{0} \boldsymbol{\tau}_{j}\right) \leq 0\right\} \\
& =\{4,8\} ;(8):=2 ;(2):=8 ;(J=(4,8,3,1,5,6,7,2,9))
\end{aligned}
$$

$k:=2$;

Step 1: Determining the elimination matrix with respect to cone $C_{2}$ :

Since ${ }^{1} \tau_{\langle 2\rangle(2)}=0$, set $\langle 2\rangle:=4,\langle 4\rangle:=2$; Doing elimination operation at eye $(2,2)$ on $\left({ }^{1} T\right)$, get $\left({ }^{2} T\right)$ :

\begin{tabular}{lrlllllllll}
\hline & $\tau_{4}$ & $\tau_{8}$ & $\tau_{3}$ & $\tau_{1}$ & $\tau_{5}$ & $\tau_{6}$ & $\tau_{7}$ & $\tau_{2}$ & $\tau_{9}$ & $\mathrm{~g}$ \\
\hline$y_{1}$ & $\mathbf{- 1}$ & 0 & 0 & 0 & 0 & 0 & 0 & 0 & 0 & -4 \\
$y_{4}$ & 0 & 1 & 0 & 0 & 0 & 0 & 0 & 0 & 0 & -6 \\
$y_{3}$ & 1 & 0 & 0 & 2 & 1 & 0 & 1 & 1 & 0 & -4 \\
$y_{2}$ & 1 & 0 & 1 & 2 & 1 & 1 & 0 & 0 & 0 & -1 \\
$y_{5}$ & 0 & 0 & 1 & 1 & 0 & 0 & 0 & 0 & 1 & -2 \\
\hline
\end{tabular}

The sub-matrix including left 2 columns form the elimination-matrix $E^{*}$ with respect to $C_{2}$.

Step 2: Getting the gradient direction $\underline{\boldsymbol{g}}_{\downarrow(1)(2)}$ at $F_{2}$ :

The solution vector of $E^{*}$ is ${ }^{k-1} \boldsymbol{\tau}_{(k)}={ }^{1} \boldsymbol{\tau}_{(2)}=(1,0,0,0,0),{ }^{1} \boldsymbol{g}=(-6,-4,-1,-2)$.

Calculate the intersection projection

$$
\begin{aligned}
& { }^{2} \boldsymbol{d}_{\downarrow}:=:=(-6,-4,-1,-2)-(-6) /(1)(1,0,0,0)=(0,-4,-1,-2) ; \\
& \underline{\boldsymbol{g}}_{\downarrow(1)(2)}:=\text { HiddenSolution }\left({ }^{2} \boldsymbol{d}_{\downarrow}\right)=(-5,0,-4,-1,-2) ;
\end{aligned}
$$

Return to original order, get $\underline{\boldsymbol{g}}_{\downarrow 4,8}=(-5,-1,-4,0,-2)$;

Step 3 Getting next stage point and the door cone $C_{3}$ :

$$
\begin{aligned}
F_{3} & =\operatorname{NextStagePoint}\left(F_{2}, \underline{\boldsymbol{g}}_{\downarrow(1)(2)}\right)=(0,5 / 2,1 / 2,0,5 / 3) \\
\Delta & \left.:=\left\{j \mid\left(F_{3},{ }^{0} \boldsymbol{\tau}_{j}\right)=c_{j}, \underline{\boldsymbol{g}}_{\downarrow(1)(2)},{ }^{0} \boldsymbol{\tau}_{j}\right) \leq 0\right\} \\
& =\{4,8,5\} ;(5):=3 ;(3):=5 ;(J=(4,8,5,1,3,6,7,2,9))
\end{aligned}
$$

$k:=3$;

Step 1: Determining the elimination matrix with respect to cone $C_{3}$ :

Do dual elimination operation at eye $(3,3)$ on $\left({ }^{2} T\right)$, get $\left({ }^{3} T\right)$ : 


\begin{tabular}{rrrrrrrrrrr}
\hline & $\tau_{4}$ & $\tau_{8}$ & $\tau_{5}$ & $\tau_{1}$ & $\tau_{3}$ & $\tau_{6}$ & $\tau_{7}$ & $\tau_{2}$ & $\tau_{9}$ & $\mathrm{~g}$ \\
\hline$y_{1}$ & $\mathbf{- 1}$ & 0 & 0 & 0 & 0 & 0 & 0 & 0 & 0 & -4 \\
$y_{4}$ & 0 & $\mathbf{1}$ & 0 & 0 & 0 & 0 & 0 & 0 & 0 & -6 \\
$y_{3}$ & 1 & 0 & $\mathbf{1}$ & 0 & 0 & 0 & 0 & 0 & 0 & -4 \\
$y_{2}$ & 1 & 0 & 1 & 0 & 1 & 1 & -1 & -1 & 0 & -1 \\
$y_{5}$ & 0 & 0 & 0 & 1 & 1 & 0 & 0 & 0 & 1 & -2 \\
\hline
\end{tabular}

The sub-matrix including left 3 columns form the elimination-matrix $E^{*}$ with respect to $C_{3}$.

Step 2: Getting the gradient direction $\underline{\boldsymbol{g}}_{\downarrow(1)(2)(3)}$ at $F_{3}$ :

The solution vector of $E^{*}$ is ${ }^{k-1} \boldsymbol{\tau}_{(k)}={ }^{2} \boldsymbol{\tau}_{(3)}=(1,1,0) .{ }^{2} \boldsymbol{g}=(-4,-1,-2)$ (In the new order of $\langle i\rangle)$. Calculate the intersection projection

$$
\begin{aligned}
& { }^{3} \boldsymbol{d}_{\downarrow}:=(-4,-1,-2)-(-5) /(2)(1,1,0)=(-3 / 2,3 / 2,-2) ; \\
& \underline{\boldsymbol{g}}_{\downarrow(1)(2)(3)}:=\text { HiddenSolution }\left({ }^{3} \boldsymbol{d}_{\downarrow}\right)=(0,0,-3 / 2,3 / 2,-2) ;
\end{aligned}
$$

Return to original order, get $\underline{\boldsymbol{g}}_{\downarrow 4,8,5}=(0,3 / 2,-3 / 2,0,-2)$;

Step 3 Getting next stage point and the door cone $C_{4}$ :

$$
\begin{aligned}
& F_{4}=\operatorname{NextStagePoint}\left(F_{3}, \underline{\boldsymbol{g}}_{\downarrow(1)(2)(3)}\right)=(0,3,0,0,1) \\
& \Delta:=\left\{j \mid\left(F_{4},{ }^{0} \boldsymbol{\tau}_{j}\right)=c_{j},\left(\underline{\boldsymbol{g}}_{\downarrow(1)(2)(3)},{ }^{0} \boldsymbol{\tau}_{j}\right) \leq 0\right\}=\{4,8,5,1\} ; \text { Existent order is ok. } \\
& \quad(J=(4,8,5,1,3,6,7,2,9))
\end{aligned}
$$

$k:=4$

Step 1: Determining the elimination matrix with respect to cone $C_{4}$ :

Since ${ }^{3} \tau_{\langle 4\rangle(4)}=0$, set $\langle 4\rangle:=5,\langle 5\rangle:=4$; Doing elimination operation at eye $(4,4)$ on $\left({ }^{3} T\right)$, get $\left({ }^{4} T\right)$ :

\begin{tabular}{rrrrrrrrrrr}
\hline & $\tau_{4}$ & $\tau_{8}$ & $\tau_{5}$ & $\tau_{1}$ & $\tau_{3}$ & $\tau_{6}$ & $\tau_{7}$ & $\tau_{2}$ & $\tau_{9}$ & $\mathrm{G}$ \\
\hline$y_{1}$ & $\mathbf{- 1}$ & 0 & 0 & 0 & 0 & 0 & 0 & 0 & 0 & -4 \\
$y_{4}$ & 0 & $\mathbf{1}$ & 0 & 0 & 0 & 0 & 0 & 0 & 0 & -6 \\
$y_{3}$ & 1 & 0 & $\mathbf{1}$ & 0 & 0 & 0 & 0 & 9 & 0 & -4 \\
$y_{5}$ & 0 & 0 & 0 & $\mathbf{1}$ & 0 & 0 & 0 & 0 & 0 & -2 \\
$y_{2}$ & 1 & 0 & 1 & 0 & 1 & 1 & -1 & -1 & 0 & -1 \\
\hline
\end{tabular}

The sub-matrix including left 4 columns form the elimination-matrix $E^{*}$ with respect to $C_{4}$.

Step 2: Getting the gradient direction $\underline{\boldsymbol{g}}_{\downarrow(1)(2)(3)(4)}$ at $F_{3}$ :

The solution vector of $E^{*}$ is ${ }^{k-1} \boldsymbol{\tau}_{(k)}={ }^{3} \boldsymbol{\tau}_{(4)}=(1,0) .{ }^{3} \boldsymbol{g}=(-2,-1)$ (In the new order of $\langle i\rangle)$.Calculate the intersection projection 


$$
\begin{aligned}
& { }^{4} \boldsymbol{d}_{\downarrow}:=(-2,-1)-(-2) /(1)(1,0)=(0,-1) ; \\
& \boldsymbol{g}_{\downarrow(1)(2)(3)(4)}:=\text { HiddenSolution }\left({ }^{4} \boldsymbol{d}_{\downarrow}\right)=(0,0,0,0,-1) ;
\end{aligned}
$$

Return to original order, get $\boldsymbol{g}_{\downarrow 4,8,5,1}=(0,-1,0,0,0)$;

$k:=5$;

Step 1: Determining the elimination matrix with respect to cone $C_{5}$ :

Doing elimination operation at eye $(5,5)$ on $\left({ }^{4} T\right)$, get $\left({ }^{5} T\right)$ :

\begin{tabular}{rrrllllllll}
\hline & $\tau_{4}$ & $\tau_{8}$ & $\tau_{5}$ & $\tau_{1}$ & $\tau_{3}$ & $\tau_{6}$ & $\tau_{7}$ & $\tau_{2}$ & $\tau_{9}$ & $\mathrm{G}$ \\
\hline$y_{1}$ & $\mathbf{- 1}$ & 0 & 0 & 0 & 0 & 0 & 0 & 0 & 0 & -4 \\
$y_{4}$ & 0 & $\mathbf{1}$ & 0 & 0 & 0 & 0 & 0 & 0 & 0 & -6 \\
$y_{3}$ & 1 & 0 & $\mathbf{1}$ & 0 & 0 & 0 & 0 & 9 & 0 & -4 \\
$y_{5}$ & 0 & 0 & 0 & $\mathbf{1}$ & 0 & 0 & 0 & 0 & 0 & -2 \\
$y_{2}$ & 1 & 0 & 1 & 0 & $\mathbf{1}$ & 0 & 0 & 0 & 0 & -1 \\
\hline
\end{tabular}

The sub-matrix including left 5 columns form the elimination-matrix $E^{*}$ with respect to $C_{5}$.

Step 2: Getting the gradient direction $\underline{g}_{\downarrow(1)(2)(3)(4)(5)}$ at $F_{5}$ :

The solution vector of $E^{*}$ is ${ }^{k-1} \boldsymbol{\tau}_{(k)}={ }^{4} \boldsymbol{\tau}_{(5)}=(1) .{ }^{4} \boldsymbol{g}=(-1)$,

$$
\begin{aligned}
& { }^{5} \boldsymbol{d}_{\downarrow}:=(-1)-(-1) /(1)(1)=(0) ; \\
& \boldsymbol{g}_{\downarrow(1)(2)(3)(4)(5)}:=\text { HiddenSolution }\left({ }^{3} \boldsymbol{d}_{\downarrow}\right)=(0,0,0,0,0) ;
\end{aligned}
$$

Return to original order, get $\boldsymbol{g}_{\downarrow 4,8,5,1,3}=(0,0,0,0,0)$;

$\boldsymbol{y}^{*}=F_{4}=(0,3,0,0,1)$ i.e., the solution of the dual LP problem is

$$
y_{1}^{*}=0, y_{2}^{*}=3, y_{3}^{*}=0, y_{4}^{*}=0, y_{5}^{*}=1 ; w^{*}=\left(V^{*}, \boldsymbol{b}\right)=5
$$

\section{Verifying:}

The simplex tableau is $\left(\underline{T}_{0}\right)$ :

$\begin{array}{ccccccccccc} & x_{1} & x_{2} & x_{3} & x_{4} & x_{5} & x_{6} & x_{7} & x_{8} & x_{9} & Z \\ x_{5} & 2 & -1 & 0 & -1 & 1 & 0 & 0 & 0 & 0 & 4 \\ x_{6} & 0 & 1 & 1 & 1 & 0 & 1 & 0 & 0 & 0 & 1 \\ x_{7} & 0 & 2 & 0 & 1 & 0 & 0 & 1 & 0 & 0 & 4 \\ x_{8} & 1 & 1 & 1 & 0 & 0 & 0 & 0 & 1 & 0 & 6 \\ x_{9} & 1 & 0 & 1 & 0 & 0 & 0 & 0 & 0 & 1 & 2 \\ & -1 & -1 & -2 & -3 & 0 & 0 & 0 & 0 & 0 & 0\end{array}$


By means of simplex's pivots, get the optimal tableau $\left(\underline{T}^{*}\right)$ :

$\begin{array}{ccccccccccc} & x_{1} & x_{2} & x_{3} & x_{4} & x_{5} & x_{6} & x_{7} & x_{8} & x_{9} & Z \\ x_{5} & 0 & 0 & -1 & 0 & 1 & 1 & 0 & 0 & -2 & 1 \\ x_{4} & 0 & 1 & 1 & 1 & 0 & 1 & 0 & 0 & 0 & 1 \\ x_{7} & 0 & 1 & 0 & 0 & 0 & -1 & 1 & 0 & 0 & 3 \\ x_{8} & 0 & 1 & 0 & 0 & 0 & 0 & 0 & 1 & -1 & 4 \\ x_{1} & 1 & 0 & 1 & 0 & 0 & 0 & 0 & 0 & 1 & 2 \\ & 0 & 2 & 2 & 0 & 0 & 3 & 0 & 0 & 1 & 5\end{array}$

The optimal solutions: $x^{*}=(2,0,0,1,1,0,3,4,0) ; y^{*}=(0,3,0,0,1,1) ; z^{*}=5$. Note. Coincidence is kept on optimal value $z^{*}=w^{*}$ only; when the optimal points are not unique, the optimal points specified may not be coincident.

The algorithm traces a broken line $\Lambda$. We do not ensure that the direction along the line has the maximal falling-gradient at all points on $\Lambda$, but we ensure that it true for all stage points on the path. The author can not prove that the track is the shortest path in $D$, but intuitively guesses that it is. If it is, then it must be a strongly polynomial-times algorithm. As a guess, hope readers give a strict proof.

\section{Discussions}

1. The projection of a vector in the intersection of a group of planes is an important topic in mathematics.

2. The methodology presented in the algorithm GradientFalling has influence not only for linear but for non-linear programming.

3. The algorithm GradientFalling guides us to have a similar algorithm in simplexvariant.

4. The paper has had big changes from the original manuscript. In the beginning, the author employees the concept of fruited cone, which inherits the idea of Chen et al. [11], to pick off the dual optimal point quickly whenever it is located on an edge of current cone. The use of fruited cone was based on such a proposition: The key angle of a cone $C$ is degenerated if and only if there is one and only one edge of $C$ lying in the key plate. Unfortunately, the proposition is wrong. Thanks Dr. Shinji Mizuno for that he points out the mistake. He gives a simple but strong anti-example as follows: Suppose that $Y=R^{4}$. Consider the intersection of the coordinate cone $C=C_{0}$ in the 2-dimensional plane

$$
\pi=\left\{\boldsymbol{y}=\left(y_{1}, y_{2}, y_{3}, y_{4}\right) \mid y_{1}=y_{2}, y_{3}+y_{4}=0\right\} .
$$

The intersection of $C$ in $\pi$ is a ray:

$$
R=C \cap \pi=\left\{\boldsymbol{y}=\left(y_{1}, y_{2}, y_{3}, y_{4}\right) \mid y_{1}=y_{2}, y_{2} \geq 0, y_{3}=0, y_{4}=0\right\} .
$$

Which is the degenerated key angle of $C$ in $\pi$. The key angle of $C_{0}$ in $\pi$ is degenerated to the ray $R$; while none of edges of $C_{0}$ lain in $\pi$. 
Since the mentioned proposition in my original manuscript was wrong, the related main algorithm ConePairCut is abandoned with fine Figs drawn by Dr. H. C. Lui.

Acknowledgments The author wishes to thank all his friends for their valuable critics, comments and assistances on this paper. Special thanks are given to Prof. Yang Xu and his team, Prof. Sicong Guo and H. T. Liu for their special reviews and checking, and to Dr. H. C. Lui for his polishing, drawing and explanation. Special thanks are also extended to his wife, Yipu Jiang, for her continuous support of this research, and to his mother school, Beijing Normal University for commemoration. This study was partially supported by the grants (Grant Nos. 61350003, 70621001, 70531040) from the Natural Science Foundation of China.

\section{References}

1. Dantzig GB (2002) Linear programming. Oper Res 50(1):42-47

2. Shi Y (2001) Multiple criteria and multiple constraint levels linear programming. World Scientific Publishing, River Edge

3. Smale S (1998) Mathematical problems for the next century. Math Intell 20(2):7-15

4. Santos F, A counterexample to the Hirsch conjecture. In: Presented at the conference 100 Years in Seattle: the mathematics of Klee and Grünbaum)

5. Zhang ZZ (1992) New algorithms for the system of linear equations and linear programming. Hong Kong Sino Tech Press, Hong Kong (In Chinese)

6. Wang PZ (2011) Cone-cutting: a variant representation of pivot in Simplex. Inf Technol Decis Mak 10(1):65-82

7. Wang PZ, Ostermark R, Alex R, Tan SH (2001) Polyhedral representation of fuzzy linear bases. Soft Comput 5:208-214

8. Pan PQ (2005) A revised dual projective pivot algorithm for linear programming. SIAM J Optim 16(1):49-68

9. Klee V, Walkup DW (1967) The d-step conjecture for polyhedra of dimension $d<6$. Acta Math 133:53-78

10. Terlaky T (2009) Algorithms and conjectures for linear optimization. In: A speech in the International Conference on Linear Programming, Hainan, China

11. Chen HD, Pardalos PM, Saunders MA (1994) The simplex algorithm with a new primal and dual pivot rule. Oper Res Lett 16(3):121-127

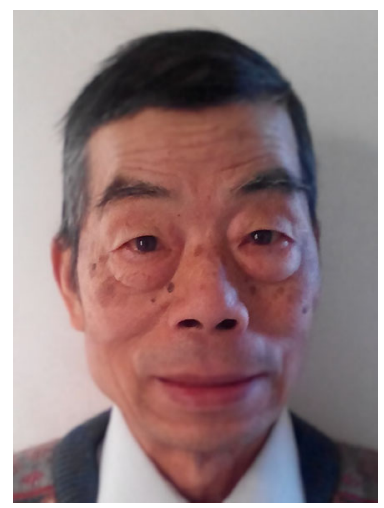

Pei-Zhuang Wang received the BS degree in Mathematics from Beijing Normal University, China in 1957. He is currently with Liaoning Technical University, His research interests include optimization and factorial analysis applied in data science. 